Article

\title{
Application of Multi-Parameter Perturbation Method to Functionally-Graded, Thin, Circular Piezoelectric Plates
}

\author{
Xiao-Ting $\mathrm{He}^{1,2, *}$, Zhi-Xin Yang ${ }^{1}$, Yang-Hui $\mathrm{Li}^{1}{ }^{1}, \mathrm{Xue} \mathrm{Li}^{1}$ and Jun-Yi Sun ${ }^{1,2}$ \\ 1 School of Civil Engineering, Chongqing University, Chongqing 400045, China; \\ 20141602063@cqu.edu.cn (Z.-X.Y.); lyhcqu@163.com (Y.-H.L.); lixuecqu@126.com (X.L.); \\ sunjunyi@cqu.edu.cn (J.-Y.S.) \\ 2 Key Laboratory of New Technology for Construction of Cities in Mountain Area (Chongqing University), \\ Ministry of Education, Chongqing 400045, China \\ * Correspondence: hexiaoting@cqu.edu.cn; Tel.: +86-(0)23-65120720
}

Received: 30 January 2020; Accepted: 1 March 2020; Published: 4 March 2020

check for updates

\begin{abstract}
In this study, a multi-parameter perturbation method is used for the solution of a functionally-graded, thin, circular piezoelectric plate. First, by assuming that elastic, piezoelectric, and dielectric coefficients of the functionally-graded materials vary in the form of the same exponential function, the basic equation expressed in terms of two stress functions and one electrical potential function are established in cylindrical coordinate system. Three piezoelectric coefficients are selected as perturbation parameters, and the established equations are solved by the multi-parameter perturbation method, thus obtaining up to first-order perturbation solutions. The validity of the perturbation solution obtained is verified by numerical simulations, based on layer-wise theory. The perturbation process indicates that adopting three piezoelectric coefficients as perturbation parameters follows the basic idea of perturbation theory-i.e., if the piezoelectricity may be regarded as a kind of introduced disturbance, the zero-order solution of the disturbance system corresponds exactly to the solution of functionally-graded plates without piezoelectricity. The result also indicates that the deformation magnitude of piezoelectric plates is smaller than that of plates without piezoelectricity, due to the well-known piezoelectric stiffening effect.
\end{abstract}

Keywords: functionally-graded piezoelectric materials; thin circular plates; multi-parameter perturbation; piezoelectric coefficients; deformation

\section{Introduction}

Piezoelectric materials have electromechanical coupling characteristics-i.e., they may generate mechanical deformation in an electric field and at the same time, electrical polarization under mechanical loads. This important characteristic makes them a good candidate for a variety of electromechanical devices-for example, sensors and actuators used extensively in electromechanical conversion [1-4]. Piezoelectric sensors are usually a laminated original made by ceramic slice. For this kind of laminated original, however, it is easy to cause stress concentration and promote the growth of interfacial microcracks, which further limits the application and development of the piezoelectric original. In order to solve this problem, functionally-graded piezoelectric materials (FGPMs), whose material properties change continuously in one direction, have been developed [5-7]. Since there is no obvious interface in this kind of material, the damage caused by the stress concentration at the interface can be avoided. This undoubtedly promotes the application of piezoelectric materials in electromechanical conversion. 
With the increasing application of functionally-graded piezoelectric materials, precise characterization of their mechanical properties is urgently needed. A great deal of research has been done on the mechanical properties of functionally-graded piezoelectric materials and structures: for example, FGPM cantilever beams [8-12], FGPM plates [13-17], and FGPM shells [18-21]. At the same time, the generation of new problems also puts forward greater requirements for the corresponding solving methods. Among all kinds of solving methods for the problem (analytical, numerical, even experimental, for example), the analytical approach is relatively challenging for the complexity of the governing equations obtained. It is known that due to electromechanical coupling, the constitutive equation becomes more complicated, compared with the purely mechanical problem in classical elasticity. The governing equation thus obtained and the variation of material properties along certain directions, due to the incorporation of functionally-graded materials, make analytical solving more and more difficult. It has been found that even for a plate consisting of classical materials, the analytical solution is hard to obtain, and in most cases, numerical simulations have to be resorted to (for example, see recent studies [22,23]). Therefore, seeking an effective analytical technique, especially since the structural property of the analytical solution may clearly show the electromechanical coupling effect in the final results, seems to be valuable and urgent. The purpose of this paper is based on this key point.

The perturbation method is one of the standard analytical methods used for the solution of nonlinear problems in applied mechanics and physics. This method consists of developing the solution of an initial or boundary value problem in an asymptotic series of a parameter, which appears explicitly either in the problem or is introduced artificially. More specifically, during the perturbation, the unknown functions are expanded in the form of ascending powers with respect to a certain small parameter. Substituting these expansions into the governing equations and corresponding boundary conditions will yield a series of equations used for determining the approximate solution of all levels by equating the same order of the perturbation parameter.

Although perturbation solutions may not rely on any small parameter, as suggested by Van Gorder [24], the perturbation parameter plays an important role in perturbation, since the right choice may permit us to obtain asymptotic solutions with better convergence. The earlier classical works may be found in Vincent's and Chien's studies. By using the external load as a perturbation parameter, Vincent [25] first obtained the perturbation solution of the Föppl-von Kármán equations. Given that the perturbation parameter either appears explicitly or is introduced artificially into the problem, Chien [26] obtained another perturbation solution using the central deflection as a perturbation parameter. Compared with experimental results, Chien's solution is accurate, and it has been cited as a classical work in subsequent studies for a long period of time. In addition to the load and the central deflection, there are several other choices for perturbation parameters-for example, a generalized displacement [27], a linear function of Poisson's ratio [28], and an average angular deflection [29]. Chen and Kuang [30] discussed the differences between the possible perturbation parameters.

In the face of the confusion of parameter selection, we usually have two entirely different ways of solving it. One is the non-parametric perturbation method proposed by Chen [31,32], in which the physical meaning of the small parameter is not given in advance, thus avoiding the empirical factors in the process of parameter selection. In other words, the selection of parameters is not constrained and is thus free, so it is also referred to as the free parameter perturbation method. Another method is the so-called "multi-parameter perturbation" method, in which two or more parameters are selected as perturbation parameters. The earlier work in this method may be found from Nowinski and Ismail [33], in which Föppl-von Kármán equations of elastic anisotropic plates were solved by selecting the anisotropy of materials and the load as two perturbation parameters. The first application of the biparametric perturbation method to beam problems was performed by Chien [34], in which the load and the height difference of end supports were selected as two perturbation parameters, and thus the classical Euler-Bernoulli equation was solved. Later, He and Chen [35] derived a biparametric perturbation solution for the same problem by simplifying the governing equation. He et al. [36] further 
studied the application of the biparametric perturbation method to beam problems with the height difference of end supports under various boundary conditions.

More recently, progress has been made in the application of this multi-parameter perturbation to bimodular plates, mainly including the combined loads problem [37], the parameter selection problem in a single load [38], and the parameter selection problem in a functionally-graded material (FGM) plate [39]. More recently, Fallah et al. [40] reviewed the perturbation method in mechanical, thermal, and thermo-mechanical loadings of cylindrical bendings of FGM plates, both clamped and simply supported, in which one- and two-parameter perturbations were used. It is worth pointing out that Lian et al. [41] used a three-parameter perturbation method to solve a FGPM cantilever beam problem for the first time, in which three piezoelectric coefficients, $D_{31}, D_{33}$, and $D_{15}$, were selected as perturbation parameters. This may be the first application of a multi-parameter perturbation in FGPM beams. Adopting piezoelectric coefficients as perturbation parameters follows the basic idea of perturbation theory, which has been reported in our previous study [42]. This basic idea indicates that so-called perturbation is essentially a kind of disturbance for an undisturbed system: if the solution of the undisturbed system may be obtained, the corresponding solution of that disturbed system may also be obtained via the perturbation technique. Given that there has been no similar application in FGPM plates, this study seems to be necessary.

In this study, we will use the multi-parameter perturbation method to solve the bending problem of a functionally-graded, thin, circular, piezoelectric plate. This paper is organized as follows. First, in Section 2, the basic equation expressed in terms of stress functions and potential function is established, in which elastic, piezoelectric, and dielectric coefficients of the functionally-graded materials vary in the form of same exponential function. By selecting three piezoelectric coefficients as perturbation parameters, in Section 3, we obtain up to first-order perturbation solution under boundary conditions. Numerical simulation and comparison with perturbation solution are given in Section 4 . The effect of the gradient index on the solution and deflections of FGPM and FGM plates are discussed in Section 5. Three important conclusions and subsequent works are summarized in Section 6.

\section{Mechanical Model and Basic Equations}

In this paper, we will study the bending problem of a functionally-graded, thin, circular, piezoelectric plate with radius $a$ and thickness $h$, as shown in Figure 1, in which the thin circular plate is subjected to uniformly-distributed external load $q$ on its upper surface, and in which the boundary conditions of its peripheries are considered to be fully fixed. Obviously, an axisymmetric deformation will generate in this plate, and it is convenient to introduce the cylindrical coordinate system to describe this problem; thus, an $r-\theta-z$ coordinate system is established, in which the coordinate origin $o$ is set at the geometric center of the plate, $r$ is the radial coordinate, $\theta$ is the circumferential coordinate (which is not shown due to axisymmetric feature), and $z$ stands for the vertical direction, as shown in Figure 1.

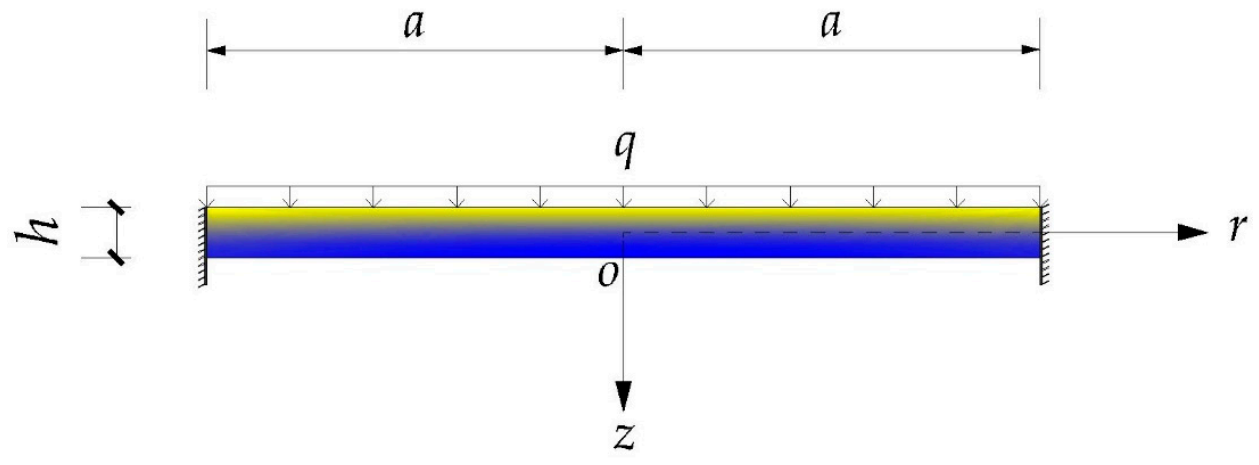

Figure 1. Scheme of a functionally-graded piezoelectric material (FGPM) thin circular plate under uniformly-distributed loads. 
Without losing generality, here we assume that the elastic coefficient $s_{i j}$, piezoelectric coefficient $d_{i j}$, and dielectric coefficient $\lambda_{i j}$ of the functionally-graded materials vary with the $z$ direction, according to the same exponential function, such that

$$
s_{i j}=s_{i j}^{0} e^{\alpha z / h}, d_{i j}=d_{i j}^{0} e^{\alpha z / h}, \lambda_{i j}=\lambda_{i j}^{0} e^{\alpha z / h},
$$

Where $\alpha$ is the functionally gradient index; and $s_{i j}^{0}, d_{i j}^{0}$, and $\lambda_{i j}^{0}$ are values of the corresponding material parameters on the middle layer $(z=0)$ of the plate, respectively.

The differential equation of equilibrium for the spatial axisymmetric problem gives

$$
\left\{\begin{array}{l}
\frac{\partial \sigma_{r}}{\partial r}+\frac{\partial \tau_{z r}}{\partial z}+\frac{\sigma_{r}-\sigma_{\theta}}{r}=0 \\
\frac{\partial \sigma_{z}}{\partial z}+\frac{\partial \tau_{r z}}{\partial r}+\frac{\tau_{r z}}{r}=0
\end{array},\right.
$$

where $\sigma_{r}, \sigma_{\theta}$, and $\sigma_{z}$ are the radial, circumferential, and axial normal stresses, respectively, and $\tau_{r z}$ is the shear stress. If we let $\varepsilon_{r}, \varepsilon_{\theta}$, and $\varepsilon_{z}$ be the radial, circumferential, and axial normal strains, respectively, and let $\gamma_{r z}$ be the shear strain, then $u_{r}$ and $u_{z}$ are the radial and axial displacement, respectively. Thus, the geometric relation of this spatial axisymmetric problem gives

$$
\left\{\begin{array}{l}
\varepsilon_{r}=\frac{\partial u_{r}}{\partial r}, \varepsilon_{\theta}=\frac{u_{r}}{r}, \\
\varepsilon_{z}=\frac{\partial u_{z}}{\partial z}, \gamma_{r z}=\frac{\partial u_{r}}{\partial z}+\frac{\partial u_{z}}{\partial r}
\end{array} .\right.
$$

The $d$-type constitutive equation of piezoelectric materials is adopted, such that

$$
\left\{\begin{array}{l}
\varepsilon_{r}=s_{11} \sigma_{r}+s_{12} \sigma_{\theta}+s_{13} \sigma_{z}+d_{31} E_{z} \\
\varepsilon_{\theta}=s_{12} \sigma_{r}+s_{11} \sigma_{\theta}+s_{13} \sigma_{z}+d_{31} E_{z} \\
\varepsilon_{z}=s_{13} \sigma_{r}+s_{13} \sigma_{\theta}+s_{33} \sigma_{z}+d_{33} E_{z} \\
\gamma_{r z}=s_{44} \tau_{r z}+d_{15} E_{r} \\
D_{r}=d_{15} \tau_{r z}+\lambda_{11} E_{r} \\
D_{z}=d_{31} \sigma_{r}+d_{31} \sigma_{\theta}+d_{33} \sigma_{z}+\lambda_{33} E_{z}
\end{array}\right.
$$

where $E_{r}$ and $E_{z}$ are the radial and axial electric field components, respectively; and $D_{r}$ and $D_{z}$ are the radial and axial electric displacement components, respectively.

In addition, the Maxwell electrical equation of equilibrium is

$$
\frac{\partial D_{r}}{\partial r}+\frac{\partial D_{z}}{\partial z}+\frac{D_{r}}{r}=0,
$$

and the relationship between the electrical field $E_{r}, E_{z}$ and the electric potential $\phi$ gives

$$
E_{r}=-\frac{\partial \phi}{\partial r}, E_{z}=-\frac{\partial \phi}{\partial z}
$$

From Equation (3), we may obtain the consistency equation of strain as follows:

$$
\left\{\begin{array}{l}
\frac{\partial^{2} \varepsilon_{r}}{\partial z^{2}}+\frac{\partial^{2} \varepsilon_{z}}{\partial r^{2}}-\frac{\partial^{2} \gamma_{r z}}{\partial r \partial z}=0 \\
\frac{\partial \varepsilon_{\theta}}{\partial r}=\frac{\varepsilon_{r}-\varepsilon_{\theta}}{r}
\end{array} .\right.
$$

Now two stress functions, $F(r, z)$ and $\psi(r, z)$, are introduced [43]; thus, we have

$$
\begin{gathered}
\sigma_{r}=\frac{\partial^{2} F}{\partial z^{2}}+\frac{1}{r} \frac{\partial \psi}{\partial r}, \sigma_{\theta}=\frac{\partial^{2} F}{\partial z^{2}}+\frac{\partial^{2} \psi}{\partial r^{2}}, \\
\sigma_{z}=\frac{1}{r} \frac{\partial}{\partial r}\left(r \frac{\partial F}{\partial r}\right), \tau_{r z}=-\frac{\partial^{2} F}{\partial r \partial z}
\end{gathered}
$$


which naturally satisfy the differential equation of equilibrium Equation (2). Substituting Equations (4), (6), and (8) into the Maxwell equation of equilibrium (Equation (5)) and consistency Equation (7), we have

$$
\begin{aligned}
& \frac{\alpha}{h} d_{33}^{0} \frac{1}{r} \frac{\partial F}{\partial r}+\frac{\alpha}{h} d_{33}^{0} \frac{\partial^{2} F}{\partial r^{2}}+\left(d_{33}^{0}-d_{15}^{0}\right) \frac{1}{r} \frac{\partial^{2} F}{\partial r \partial z}+\frac{2 \alpha}{h} d_{31}^{0} \frac{\partial^{2} F}{\partial z^{2}}+2 d_{31}^{0} \frac{\partial^{3} F}{\partial z^{3}} \\
& +\left(d_{33}^{0}-d_{15}^{0}\right) \frac{\partial^{3} F}{\partial r^{2} \partial z}+\frac{\alpha}{h} d_{31}^{0} \frac{1}{r} \frac{\partial \psi}{\partial r}+\frac{\alpha}{h} d_{31}^{0} \frac{\partial^{2} \psi}{\partial r^{2}}+d_{31}^{0} \frac{1}{r} \frac{\partial^{2} \psi}{\partial r \partial z}+d_{31}^{0} \frac{\frac{\partial}{3} \psi}{\partial r^{2} \partial z} \\
& =\lambda_{11}^{0} \frac{1}{r} \frac{\partial \phi}{\partial r}+\frac{\alpha}{h} \lambda_{33}^{0} \frac{\partial \phi}{\partial z}+\lambda_{11}^{0} \frac{\partial^{2} \phi}{\partial r^{2}}+\lambda_{33}^{0} \frac{\partial^{2} \phi}{\partial z^{2}} \\
& \left(\frac{\alpha^{2}}{h^{2}} s_{13}^{0}+s_{33}^{0} \frac{2}{r^{2}}\right) \frac{1}{r} \frac{\partial F}{\partial r}+\left(\frac{\alpha^{2}}{h^{2}} s_{13}^{0}-s_{33}^{0} \frac{2}{r^{2}}\right) \frac{\partial^{2} F}{\partial r^{2}}+\frac{2 \alpha}{h} s_{13}^{0} \frac{1}{r} \frac{\partial^{2} F}{\partial r \partial z} \\
& +\frac{\alpha^{2}}{h^{2}}\left(s_{11}^{0}+s_{12}^{0}\right) \frac{\partial^{2} F}{\partial z^{2}}+s_{33}^{0} \frac{1}{r} \frac{\partial^{3} F}{\partial r^{3}}+\frac{\alpha}{h}\left(2 s_{13}^{0}+s_{44}^{0}\right) \frac{\partial^{3} F}{\partial r^{2} \partial z}+s_{13}^{0} \frac{1}{r} \frac{\partial^{3} F}{\partial r \partial z^{2}} \\
& +\frac{2 \alpha}{h}\left(s_{11}^{0}+s_{12}^{0}\right) \frac{\partial^{3} F}{\partial z^{3}}+s_{33}^{0} \frac{\partial^{4} F}{\partial r^{4}}+\left(3 s_{13}^{0}+s_{44}^{0}\right) \frac{\partial^{4} F}{\partial r^{2} \partial z^{2}}+\left(s_{11}^{0}+s_{12}^{0}\right) \frac{\partial^{4} F}{\partial z^{4}} \\
& +\left(\frac{\alpha^{2}}{h^{2}} s_{11}^{0}+s_{13}^{0} \frac{2}{r^{2}}\right) \frac{1}{r} \frac{\partial \psi}{\partial r}+\left(\frac{\alpha^{2}}{h^{2}} s_{12}^{0}-s_{13}^{0} \frac{2}{r^{2}}\right) \frac{\partial^{2} \psi}{\partial r^{2}}+\frac{2 \alpha}{h} s_{11}^{0} \frac{1}{r} \frac{\partial^{2} \psi}{\partial r \partial z} \\
& +s_{13}^{0} \frac{1}{r} \frac{\partial^{3} \psi}{\partial r^{3}}+\frac{2 \alpha}{h} s_{12}^{0} \frac{\partial^{3} \psi}{\partial r^{2} \partial z}+s_{11}^{0} \frac{1}{r} \frac{\partial^{3} \psi}{\partial r \partial z^{2}}+s_{13}^{0} \frac{\partial^{4} \psi}{\partial r^{4}}+s_{12}^{0} \frac{\partial^{2} \psi}{\partial r^{2} \partial z^{2}} \\
& =\frac{\alpha^{2}}{h^{2}} d_{31}^{0} \frac{\partial \phi}{\partial z}-\frac{\alpha}{h} d_{15}^{0} \frac{\partial^{2} \phi}{\partial r^{2}}+\frac{2 \alpha}{h} d_{31}^{0} \frac{\partial^{2} \phi}{\partial z^{2}}-\left(d_{15}^{0}-d_{33}^{0}\right) \frac{\partial^{3} \phi}{\partial r^{2} \partial z}+d_{31}^{0} \frac{\partial^{3} \phi}{\partial z^{3}}
\end{aligned}
$$

and

$$
\begin{aligned}
& -s_{13}^{0} \frac{1}{2} \frac{\partial F}{\partial r}+s_{13}^{0} \frac{1}{r} \frac{\partial^{2} F}{\partial r^{2}}+s_{13}^{0} \frac{\partial^{3} F}{\partial r^{3}}+\left(s_{11}^{0}+s_{12}^{0}\right) \frac{\partial^{3} F}{\partial r \partial z^{2}}-s_{11}^{0} \frac{1}{r^{2}} \frac{\partial \psi}{\partial r} \\
& +s_{11}^{0} \frac{1}{r} \frac{\partial^{2} \psi}{\partial r^{2}}+s_{11}^{0} \frac{\partial^{3} \psi}{\partial r^{3}}-d_{31}^{0} \frac{\partial^{2} \phi}{\partial r \partial z}=0
\end{aligned}
$$

Thus, we obtain the three governing equations expressed in terms of $F(r, z), \psi(r, z)$, and $\phi(r, z)$. The boundary conditions are considered as follows: for the upper surface of the plate

$$
\sigma_{z}=-q, \tau_{r z}=0, D_{z}=0, \text { at } z=-\frac{h}{2}
$$

and for the lower surface of the plate

$$
\sigma_{z}=0, \tau_{r z}=0, D_{z}=0, \text { at } z=\frac{h}{2}
$$

In addition, the displacement condition at the fixed periphery is considered to be

$$
u_{z}=0, u_{r}=0, \frac{\partial u_{z}}{\partial r}=0, \text { at } r=a \text { and } z=z_{n},
$$

where $z_{n}$ is the location of the neutral layer of the bending plate, and is temporarily unknown.

\section{Application of Multi-Parameter Perturbation Method}

\subsection{Nondimensionalization and Perturbation Expansions}

We introduce the following dimensionless quantities:

$$
\begin{gathered}
\beta=\frac{r}{h}, \eta=\frac{z}{h}, D_{15}=\frac{d_{15}^{0}}{\sqrt{s_{11}^{0} \lambda_{11}^{0}}}, D_{33}=\frac{d_{33}^{0}}{\sqrt{s_{11}^{0} \lambda_{11}^{0}}}, D_{31}=\frac{d_{31}^{0}}{\sqrt{s_{11}^{0} \lambda_{11}^{0}}}, \\
S_{12}=\frac{s_{12}^{0}}{s_{11}^{0}}, S_{13}=\frac{s_{13}^{0}}{s_{11}^{0}}, S_{33}=\frac{s_{33}^{0}}{s_{11}^{0}}, S_{44}=\frac{s_{44}^{0}}{s_{11}^{0}}, \bar{\lambda}_{33}=\frac{\lambda_{33}^{0}}{\lambda_{11}^{0}}, \\
\bar{F}=\frac{F s_{11}^{0}}{h^{2}}, \bar{\psi}=\frac{\psi s_{11}^{0}}{h^{2}}, \bar{\phi}=\frac{\phi \sqrt{s_{11}^{0} \lambda_{11}^{0}}}{h},
\end{gathered}
$$

and

$$
\begin{gathered}
\bar{q}=s_{11}^{0} q, \bar{\sigma}_{r}=s_{11}^{0} \sigma_{r}, \bar{\sigma}_{\theta}=s_{11}^{0} \sigma_{\theta}, \bar{\sigma}_{z}=s_{11}^{0} \sigma_{z}, \bar{\tau}_{r z}=s_{11}^{0} \tau_{r z}, \bar{u}_{r}=\frac{u_{r}}{h}, \bar{u}_{z}=\frac{u_{z}}{h}, \\
\bar{D}_{r}=D_{r} \sqrt{s_{11}^{0} / \lambda_{11}^{0}}, \bar{D}_{z}=D_{z} \sqrt{s_{11}^{0} / \lambda_{11}^{0}}, \bar{E}_{r}=E_{r} \sqrt{s_{11}^{0} \lambda_{11}^{0}}, \bar{E}_{z}=E_{z} \sqrt{s_{11}^{0} \lambda_{11}^{0}} .
\end{gathered}
$$


where Equation (15a) is used for the nondimensionalization of governing equations, and Equation $(15 b)$ is only for later computation. Substituting the above dimensionless quantities into the three governing equations, (9), (10), and (11), and for the convenience of writing and reading, also replacing these dimensionless quantities

$$
\bar{q}, \bar{\sigma}_{r}, \bar{\sigma}_{\theta}, \bar{\sigma}_{z}, \bar{\tau}_{r z}, \bar{D}_{r}, \bar{D}_{z}, \bar{E}_{r}, \bar{E}_{z}, \bar{\lambda}_{33}, \bar{u}_{r}, \bar{u}_{z}, \bar{F}, \bar{\psi}, \bar{\phi}
$$

with the original forms, respectively,

$$
q, \sigma_{r}, \sigma_{\theta}, \sigma_{z}, \tau_{r z}, D_{r}, D_{z}, E_{r}, E_{z}, \lambda_{33}, u_{r}, u_{z}, F, \psi, \phi,
$$

we finally obtain

$$
\begin{aligned}
& \alpha D_{33} \frac{\partial F}{\partial \beta}+\alpha \beta D_{33} \frac{\partial^{2} F}{\partial \beta^{2}}+\left(D_{33}-D_{15}\right) \frac{\partial^{2} F}{\partial \beta \partial \eta}+2 \alpha \beta D_{31} \frac{\partial^{2} F}{\partial \eta^{2}}+\beta\left(D_{33}-D_{15}\right) \frac{\partial^{3} F}{\partial \beta^{2} \partial \eta} \\
& +2 \beta D_{31} \frac{\partial^{3} F}{\partial \eta^{3}}+\alpha D_{31} \frac{\partial \psi}{\partial \beta}+\alpha \beta D_{31} \frac{\partial^{2} \psi}{\partial \beta^{2}}+D_{31} \frac{\partial^{2} \psi}{\partial \beta \partial \eta}+\beta D_{31} \frac{\partial^{3} \psi}{\partial \beta^{2} \partial \eta}-\frac{\partial \phi}{\partial \beta}-\alpha \beta \lambda_{33} \frac{\partial \phi}{\partial \eta} \\
& -\beta \frac{\partial^{2} \phi}{\partial \beta^{2}}-\beta \lambda_{33} \frac{\partial^{2} \phi}{\partial \eta^{2}}=0 \\
& \left(\alpha^{2} \beta^{2} S_{13}+2 S_{33}\right) \frac{\partial F}{\partial \beta}+\left(\alpha^{2} \beta^{3} S_{13}-2 \beta S_{33}\right) \frac{\partial^{2} F}{\partial \beta^{2}}+2 \alpha \beta^{2} S_{13} \frac{\partial^{2} F}{\partial \beta \partial \eta}+\alpha^{2} \beta^{3}\left(1+S_{12}\right) \\
& \frac{\partial^{2} F}{\partial \eta^{2}}+2 \alpha \beta^{3}\left(1+S_{12}\right) \frac{\partial^{3} F}{\partial \eta^{3}}+\alpha \beta^{3}\left(2 S_{13}+S_{44}\right) \frac{\partial^{3} F}{\partial \beta^{2} \partial \eta}+\beta^{2} S_{33} \frac{\partial^{3} F}{\partial \beta^{3}}+\beta^{2} S_{13} \frac{\partial^{3} F}{\partial \beta \partial \eta^{2}} \\
& +\beta^{3} S_{33} \frac{\partial^{4} F}{\partial \beta^{4}}+\beta^{3}\left(3 S_{13}+S_{44}\right) \frac{\partial^{4} F}{\partial \beta^{2} \partial \eta^{2}}+\beta^{3}\left(1+S_{12}\right) \frac{\partial^{4} F}{\partial \eta^{4}}+\left(\alpha^{2} \beta^{2}+2 S_{13}\right) \frac{\partial \psi}{\partial \beta} \\
& +\left(\alpha^{2} \beta^{3} S_{12}-2 \beta S_{13}\right) \frac{\partial^{2} \psi}{\partial \beta^{2}}+2 \alpha \beta^{2} \frac{\partial^{2} \psi}{\partial \beta \partial \eta}+\beta^{2} S_{13} \frac{\partial^{3} \psi}{\partial \beta^{3}}+2 \alpha \beta^{3} S_{12} \frac{\partial^{3} \psi}{\partial \beta^{2} \partial \eta}+\beta^{2} \frac{\partial^{3} \psi}{\partial \beta \partial \eta^{2}} \\
& +\beta^{3} S_{13} \frac{\partial^{4} \psi}{\partial \beta^{4}}+\beta^{3} S_{12} \frac{\partial^{4} \psi}{\partial \beta^{2} \partial \eta^{2}}-\alpha^{2} \beta^{3} D_{31} \frac{\partial \phi}{\partial \eta}+\alpha \beta^{3} D_{15} \frac{\partial^{2} \phi}{\partial \beta^{2}}-2 \alpha \beta^{3} D_{31} \frac{\partial^{2} \phi}{\partial \eta^{2}} \\
& +\beta^{3}\left(D_{15}-D_{33}\right) \frac{\partial^{3} \phi}{\partial \beta^{2} \partial \eta}-\beta^{3} D_{31} \frac{\partial^{3} \phi}{\partial \eta^{3}}=0
\end{aligned}
$$

and

$$
\begin{aligned}
& -S_{13} \frac{\partial F}{\partial \beta}+\beta S_{13} \frac{\partial^{2} F}{\partial \beta^{2}}+\beta^{2} S_{13} \frac{\partial^{3} F}{\partial \beta^{3}}+\beta^{2}\left(1+S_{12}\right) \frac{\partial^{3} F}{\partial \beta \partial \eta^{2}}-\frac{\partial \psi}{\partial \beta}+\beta \frac{\partial^{2} \psi}{\partial \beta^{2}}+\beta^{2} \frac{\partial^{3} \psi}{\partial \beta^{3}} \\
& -\beta^{2} D_{31} \frac{\partial^{2} \phi}{\partial \beta \partial \eta}=0
\end{aligned}
$$

In the above three equations, $F=F(\beta, \eta), \psi=\psi(\beta, \eta)$, and $\phi=\phi(\beta, \eta)$ are dimensionless functions; $S_{i j}, D_{i j}$, and $\lambda_{i j}$ are dimensionless constants. If there is no special explanation below, the next symbols in this paper refer to dimensionless quantities. We select the zero point values of three piezoelectric coefficients, $D_{31}, D_{33}$, and $D_{15}$, as our perturbation parameters, and expand the unknown functions, $F, \psi$, and $\phi$, with respect to the parameters:

$$
\left\{\begin{array}{c}
F=F_{1}^{(0)}+F_{1}^{(1)} D_{31}+F_{2}^{(1)} D_{33}+F_{3}^{(1)} D_{15}+F_{1}^{(2)} D_{31}^{2}+F_{2}^{(2)} D_{33}^{2} \\
+F_{3}^{(2)} D_{15}^{2}+F_{4}^{(2)} D_{31} D_{33}+F_{5}^{(2)} D_{31} D_{15}+F_{6}^{(2)} D_{33} D_{15}+\cdots \cdots \\
\psi=\psi_{1}^{(0)}+\psi_{1}^{(1)} D_{31}+\psi_{2}^{(1)} D_{33}+\psi_{3}^{(1)} D_{15}+\psi_{1}^{(2)} D_{31}^{2}+\psi_{2}^{(2)} D_{33}^{2} \\
+\psi_{3}^{(2)} D_{15}^{2}+\psi_{4}^{(2)} D_{31} D_{33}+\psi_{5}^{(2)} D_{31} D_{15}+\psi_{6}^{(2)} D_{33} D_{15}+\cdots \cdots \\
\phi=\phi_{1}^{(0)}+\phi_{1}^{(1)} D_{31}+\phi_{2}^{(1)} D_{33}+\phi_{3}^{(1)} D_{15}+\phi_{1}^{(2)} D_{31}^{2}+\phi_{2}^{(2)} D_{33}^{2} \\
+\phi_{3}^{(2)} D_{15}^{2}+\phi_{4}^{(2)} D_{31} D_{33}+\phi_{5}^{(2)} D_{31} D_{15}+\phi_{6}^{(2)} D_{33} D_{15}+\cdots \cdots
\end{array}\right.
$$

We also let

$$
\left\{\begin{array}{l}
F_{j}^{(i)}(\beta, \eta)=\beta^{4} f_{j 1}^{(i)}(\eta)+\beta^{2} f_{j 2}^{(i)}(\eta)+f_{j 3}^{(i)}(\eta), \\
\psi_{j}^{(i)}(\beta, \eta)=\beta^{4} g_{j 1}^{(i)}(\eta)+\beta^{2} g_{j 2}^{(i)}(\eta), \\
\phi_{j}^{(i)}(\beta, \eta)=\beta^{4} h_{j 1}^{(i)}(\eta)+\beta^{2} h_{j 2}^{(i)}(\eta)+h_{j 3}^{(i)}(\eta)
\end{array},\right.
$$


where $F_{j}^{(i)}(\beta, \eta), \psi_{j}^{(i)}(\beta, \eta)$, and $\phi_{j}^{(i)}(\beta, \eta)$ are the $j$-th functions with respect to the $i$-th order of perturbation parameters; and $f_{j 1}^{(i)}(\eta), f_{j 2}^{(i)}(\eta), f_{j 3}^{(i)}(\eta), g_{j 1}^{(i)}(\eta), g_{j 2}^{(i)}(\eta), h_{j 1}^{(i)}(\eta), h_{j 2}^{(i)}(\eta)$, and $h_{j 3}^{(i)}(\eta)$ are the component functions. It should be noted here that the expansion pattern of $F_{j}^{(i)}(\beta, \eta), \psi_{j}^{(i)}(\beta, \eta)$, and $\phi_{j}^{(i)}(\beta, \eta)$-i.e., Equation (20)—is consistent with the pattern of a similar beam problem [42]. In addition, we found that the odd power term of $\beta$ disappears in the later derivation, and only even power terms remain. For the stress function $\psi(\beta, \eta), \beta^{0}$ term will disappear in the later stress derivations (easily seen from Equation (8)); thus, there is no need to list this term in the expansion of $\psi(\beta, \eta)$.

\subsection{Zero-Order Perturbation Solution}

Substituting Equation (19) into Equations (16)-(18), we firstly have the zero-order perturbation equations as follows:

$$
\begin{gathered}
\frac{\partial \phi_{1}^{(0)}}{\partial \beta}+\alpha \beta \lambda_{33} \frac{\partial \phi_{1}^{(0)}}{\partial \eta}+\beta \frac{\partial^{2} \phi_{1}^{(0)}}{\partial \beta^{2}}+\beta \lambda_{33} \frac{\partial^{2} \phi_{1}^{(0)}}{\partial \eta^{2}}=0 \\
\left(\alpha^{2} \beta^{2} S_{13}+2 S_{33}\right) \frac{\partial F_{1}^{(0)}}{\partial \beta}+\left(\alpha^{2} \beta^{3} S_{13}-2 \beta S_{33}\right) \frac{\partial^{2} F_{1}^{(0)}}{\partial \beta^{2}}+\beta^{2} S_{33} \frac{\partial^{3} F_{1}^{(0)}}{\partial \beta^{3}}+2 \alpha \beta^{2} S_{13} \\
\frac{\partial^{2} F_{1}^{(0)}}{\partial \beta \partial \eta}+\alpha^{2} \beta^{3}\left(1+S_{12}\right) \frac{\partial^{2} F_{1}^{(0)}}{\partial \eta^{2}}+\alpha \beta^{3}\left(2 S_{13}+S_{44}\right) \frac{\partial^{3} F_{1}^{(0)}}{\partial \beta^{2} \eta \eta}+\beta^{2} S_{13} \frac{\partial^{3} F_{1}^{(0)}}{\partial \beta \partial \eta^{2}}+2 \alpha \beta^{3} \\
\left(1+S_{12}\right) \frac{\partial^{3} F_{1}^{(0)}}{\partial \eta^{3}}+\beta^{3}\left(3 S_{13}+S_{44}\right) \frac{\partial^{4} F_{1}^{(0)}}{\partial \beta^{2} \partial \eta^{2}}+\beta^{3} S_{33} \frac{\partial^{4} F_{1}^{(0)}}{\partial \beta^{4}}+\beta^{3}\left(1+S_{12}\right) \frac{\partial^{4} F_{1}^{(0)}}{\partial \eta^{4}} \\
+\left(\alpha^{2} \beta^{3} S_{12}-2 \beta S_{13}\right) \frac{\partial^{2} \psi_{1}^{(0)}}{\partial \beta^{2}}+\left(\alpha^{2} \beta^{2}+2 S_{13}\right) \frac{\partial \psi_{1}^{(0)}}{\partial \beta}+2 \alpha \beta^{2} \frac{\partial^{2} \psi_{1}^{(0)}}{\partial \beta \partial \eta}+\beta^{2} S_{13} \frac{\partial^{3} \psi_{1}^{(0)}}{\partial \beta^{3}} \\
+2 \alpha \beta^{3} S_{12} \frac{\partial^{3} \psi_{1}^{(0)}}{\partial \beta^{2} \partial \eta}+\beta^{2} \frac{\partial^{3} \psi_{1}^{(0)}}{\partial \beta \partial \eta^{2}}+\beta^{3} S_{13} \frac{\partial^{4} \psi_{1}^{(0)}}{\partial \beta^{4}}+\beta^{3} S_{12} \frac{\partial^{4} \psi_{1}^{(0)}}{\partial \beta^{2} \partial \eta^{2}}=0
\end{gathered}
$$

and

$$
\begin{aligned}
& -S_{13} \frac{\partial F_{1}^{(0)}}{\partial \beta}+\beta S_{13} \frac{\partial^{2} F_{1}^{(0)}}{\partial \beta^{2}}+\beta^{2}\left(1+S_{12}\right) \frac{\partial^{3} F_{1}^{(0)}}{\partial \beta \partial \eta^{2}}+\beta^{2} S_{13} \frac{\partial^{3} F_{1}^{(0)}}{\partial \beta^{3}}-\frac{\partial \psi_{1}^{(0)}}{\partial \beta} \\
& +\beta \frac{\partial^{2} \psi_{1}^{(0)}}{\partial \beta^{2}}+\beta^{2} \frac{\partial^{3} \psi_{1}^{(0)}}{\partial \beta^{3}}=0
\end{aligned}
$$

Substituting Equation (20) into Equations (21)-(23), we obtain the following three ordinary differential equations:

$$
\begin{aligned}
& \beta^{5}\left(\lambda_{33} \frac{d^{2} h_{11}^{(0)}}{d \eta^{2}}+\alpha \lambda_{33} \frac{d h_{11}^{(0)}}{d \eta}\right)+\beta^{3}\left(16 h_{11}^{(0)}+\lambda_{33} \frac{d^{2} h_{12}^{(0)}}{d \eta^{2}}+\alpha \lambda_{33} \frac{d h_{12}^{(0)}}{d \eta}\right)+\beta\left(4 h_{12}^{(0)}\right. \\
&\left.+\lambda_{33} \frac{d^{2} h_{13}^{(0)}}{d \eta^{2}}+\alpha \lambda_{33} \frac{d h_{13}^{(0)}}{d \eta}\right)=0 \\
& \beta^{3}\left[32 S_{33} f_{11}^{(0)}+4 \alpha^{2} S_{13} f_{12}^{(0)}+\alpha\left(8 S_{13}+2 S_{44}\right) \frac{d f_{12}^{(0)}}{d \eta}+\alpha^{2}\left(1+S_{12}\right) \frac{d^{2} f_{13}^{(0)}}{d \eta^{2}}\right. \\
&+\left(8 S_{13}+2 S_{44}\right) \frac{d^{2} f_{12}^{(0)}}{d \eta^{2}}+\alpha\left(2+2 S_{12}\right) \frac{d^{3} f_{13}^{(0)}}{d \eta^{3}}+\left(1+S_{12}\right) \frac{d^{4} f_{13}^{(0)}}{d \eta^{4}}+32 S_{13} g_{11}^{(0)} \\
&\left.+\alpha^{2}\left(2+2 S_{12}\right) g_{12}^{(0)}+\alpha\left(4+4 S_{12}\right) \frac{d g_{12}^{(0)}}{d \eta}+\left(2+2 S_{12}\right) \frac{d^{2} g_{12}^{(0)}}{d \eta^{2}}\right] \\
&+\beta^{5}\left[16 \alpha^{2} S_{13} f_{11}^{(0)}+\alpha\left(32 S_{13}+12 S_{44}\right) \frac{d f_{11}^{(0)}}{d \eta}+\left(40 S_{13}+12 S_{44}\right) \frac{d^{2} f_{11}^{(0)}}{d \eta^{2}}\right. \\
&+\alpha^{2}\left(1+S_{12}\right) \frac{d^{2} f_{12}^{(0)}}{d \eta^{2}}+\alpha\left(2+2 S_{12}\right) \frac{d^{3} f_{12}^{(0)}}{d \eta^{3}}+\left(1+S_{12}\right) \frac{d^{4} f_{12}^{(0)}}{d \eta^{4}}+\alpha^{2}\left(4+12 S_{12}\right) g_{11}^{(0)} \\
&\left.+\alpha\left(8+24 S_{12}\right) \frac{d g_{11}^{(0)}}{d \eta}+\left(4+12 S_{12}\right) \frac{d^{2} g_{11}^{(0)}}{d \eta^{2}}\right] \\
&+ \beta^{7}\left[\alpha^{2}\left(1+S_{12}\right) \frac{d_{11}^{2} f_{11}^{(0)}}{d \eta^{2}}+\alpha\left(2+2 S_{12}\right) \frac{d_{11}^{3} f_{11}^{(0)}}{d \eta^{3}}+\left(1+S_{12}\right) \frac{d^{4} f_{11}^{(0)}}{d \eta^{4}}\right]=0
\end{aligned}
$$


and

$$
\beta^{3}\left[32 S_{13} f_{11}^{(0)}+\left(2+2 S_{12}\right) \frac{d^{2} f_{12}^{(0)}}{d \eta^{2}}+32 g_{11}^{(0)}\right]+\beta^{5}\left[\left(4+4 S_{12}\right) \frac{d^{2} f_{11}^{(0)}}{d \eta^{2}}\right]=0,
$$

where $f_{11}^{(0)}(\eta), f_{12}^{(0)}(\eta), f_{13}^{(0)}(\eta), g_{11}^{(0)}(\eta), g_{12}^{(0)}(\eta), h_{11}^{(0)}(\eta), h_{12}^{(0)}(\eta)$, and $h_{13}^{(0)}(\eta)$ are unknown component functions of the zero-order perturbation. The satisfaction for the above equations will give the conditions that the same power term of $\beta$ are uniformly zero. Thus, we obtain

$$
\begin{gathered}
\left\{\begin{array}{l}
\beta^{5}: \lambda_{33} \frac{d^{2} h_{11}^{(0)}}{d \eta^{2}}+\alpha \lambda_{33} \frac{d h_{11}^{(0)}}{d \eta}=0 \\
\beta^{3}: 16 h_{11}^{(0)}+\lambda_{33} \frac{d^{2} h_{12}^{(0)}}{d \eta^{2}}+\alpha \lambda_{33} \frac{d h_{12}^{(0)}}{d \eta}=0 \\
\beta: 4 h_{12}^{(0)}+\lambda_{33} \frac{d^{2} h_{13}^{(0)}}{d \eta^{2}}+\alpha \lambda_{33} \frac{d h_{13}^{(0)}}{d \eta}=0
\end{array}\right. \\
\left\{\begin{array}{l}
\beta^{3}: 32 S_{33} f_{11}^{(0)}+4 \alpha^{2} S_{13} f_{12}^{(0)}+\alpha\left(8 S_{13}+2 S_{44}\right) \frac{d f_{12}^{(0)}}{d \eta}+\left(8 S_{13}+2 S_{44}\right) \frac{d^{2} f_{12}^{(0)}}{d \eta^{2}} \\
+\alpha^{2}\left(1+S_{12}\right) \frac{d^{2} f_{13}^{(0)}}{d \eta^{2}}+32 S_{13} g_{11}^{(0)}+\alpha\left(2+2 S_{12}\right) \frac{d^{3} f_{13}^{(0)}}{d \eta^{3}}+\left(1+S_{12}\right) \frac{d^{4} f_{13}^{(0)}}{d \eta^{4}} \\
+\alpha^{2}\left(2+2 S_{12}\right) g_{12}^{(0)}+\alpha\left(4+4 S_{12}\right) \frac{d g_{12}^{(0)}}{d \eta}+\left(2+2 S_{12}\right) \frac{d^{2} g_{12}^{(0)}}{d \eta^{2}}=0 \\
\beta^{5}: 16 \alpha^{2} S_{13} f_{11}^{(0)}+\alpha\left(32 S_{13}+12 S_{44}\right) \frac{d f_{11}^{(0)}}{d \eta}+\left(40 S_{13}+12 S_{44}\right) \frac{d^{2} f_{11}^{(0)}}{d \eta^{2}} \\
+\alpha^{2}\left(1+S_{12}\right) \frac{d^{2} f_{12}^{(0)}}{d \eta^{2}}+\alpha\left(2+2 S_{12}\right) \frac{d^{3} f_{12}^{(0)}}{d \eta^{3}}+\left(1+S_{12}\right) \frac{d^{4} f_{12}^{(0)}}{d \eta^{4}} \\
+\alpha^{2}\left(4+12 S_{12}\right) g_{11}^{(0)}+\alpha\left(8+24 S_{12}\right) \frac{d g_{11}^{(0)}}{d \eta}+\left(4+12 S_{12}\right) \frac{d^{2} g_{11}^{(0)}}{d \eta^{2}}=0 \\
\beta^{7}: \alpha^{2}\left(1+S_{12}\right) \frac{d^{2} f_{11}^{(0)}}{d \eta^{2}}+\alpha\left(2+2 S_{12}\right) \frac{d^{3} f_{11}^{(0)}}{d \eta^{3}}+\left(1+S_{12}\right) \frac{d^{4} f_{11}^{(0)}}{d \eta^{4}}=0
\end{array}\right.
\end{gathered}
$$

and

$$
\left\{\begin{array}{l}
\beta^{3}: 32 S_{13} f_{11}^{(0)}+\left(2+2 S_{12}\right) \frac{d^{2} f_{12}^{(0)}}{d \eta^{2}}+32 g_{11}^{(0)}=0 \\
\beta^{5}:\left(4+4 S_{12}\right) \frac{d^{2} f_{11}^{(0)}}{d \eta^{2}}=0
\end{array} .\right.
$$

Solving Equations (27)-(29), we have

$$
\left\{\begin{array}{l}
f_{11}^{(0)}=A_{1}^{(0)} \eta+A_{2}^{(0)}, f_{12}^{(0)}=-\frac{16 e^{-\alpha \eta}}{\left(1+S_{12}\right.}\left[\frac{1}{\alpha^{2}} B_{1}^{(0)}+\left(\frac{1}{\alpha^{2}} \eta+\frac{2}{\alpha^{3}}\right) B_{2}^{(0)}\right]+A_{3}^{(0)} \eta+A_{4}^{(0)}, \\
g_{11}^{(0)}=\left(B_{1}^{(0)}+B_{2}^{(0)} \eta\right) e^{-\alpha \eta}, h_{11}^{(0)}=C_{1}^{(0)}+C_{2}^{(0)} e^{-\alpha \eta}, \\
h_{12}^{(0)}=C_{3}^{(0)}+C_{4}^{(0)} e^{-\alpha \eta}-\frac{16 C_{1}^{(0)}}{\alpha \lambda_{33}} \eta+\frac{16 C_{2}^{(0)}}{\alpha \lambda_{33}} \eta e^{-\alpha \eta}, \\
h_{13}^{(0)}=C_{5}^{(0)}+C_{6}^{(0)} e^{-\alpha \eta}-\frac{4 C_{3}^{(0)}}{\alpha \lambda_{33}} \eta+\frac{4 C_{4}^{(0)}}{\alpha \lambda_{33}} \eta e^{-\alpha \eta} \\
+\eta\left(\frac{32 C_{1}^{(0)}}{\alpha^{2} \lambda_{33}^{2}} \eta-\frac{64 C_{1}^{(0)}}{\alpha^{3} \lambda_{33}^{2}}\right)+\eta\left(\frac{32 C_{2}^{(0)}}{\alpha^{2} \lambda_{33}^{2}} \eta+\frac{64 C_{2}^{(0)}}{\alpha^{3} \lambda_{33}^{2}}\right) e^{-\alpha \eta} \\
\frac{d^{2} f_{13}^{(0)}}{d \eta^{2}}+2 g_{12}^{(0)}=\frac{1}{\left(1+S_{12}\right)}\left\{\left[\frac{16 S_{13}\left(1-S_{12}\right)}{3\left(1+S_{12}\right)} B_{2}^{(0)} \eta^{3}+\frac{16 S_{13}\left(1-S_{12}\right)}{\left(1+S_{12}\right)} B_{1}^{(0)} \eta^{2}\right.\right. \\
\left.\left.\quad-\frac{16 S_{44}}{\alpha\left(1+S_{12}\right)} B_{2}^{(0)} \eta^{2}+A_{5}^{(0)}+A_{6}^{(0)} \eta\right] e^{-\alpha \eta}-4 S_{13} A_{3}^{(0)} \eta-4 S_{13} A_{4}^{(0)}-\frac{2 S_{44}}{\alpha} A_{3}^{(0)}\right\} .
\end{array}\right.
$$

in which $A_{1}^{(0)}, A_{2}^{(0)}, A_{3}^{(0)}, A_{4}^{(0)}, A_{5}^{(0)}, A_{6}^{(0)}, B_{1}^{(0)}, B_{2}^{(0)}, C_{1}^{(0)}, C_{2}^{(0)}, C_{3}^{(0)}, C_{4}^{(0)}, C_{5}^{(0)}$, and $C_{6}^{(0)}$ are integration constants of the zero-order perturbation, and their determination will use the corresponding boundary conditions.

The boundary conditions used for the zero-order perturbation solution are, for the upper surface:

$$
\frac{1}{\beta} \frac{\partial F_{1}^{(0)}}{\partial \beta}+\frac{\partial^{2} F_{1}^{(0)}}{\partial \beta^{2}}=-q,-\frac{\partial^{2} F_{1}^{(0)}}{\partial \beta \partial \eta}=0, \lambda_{33}\left(-\frac{\partial \phi_{1}^{(0)}}{\partial \eta}\right)=0, \text { at } \eta=-\frac{1}{2} ;
$$


for the lower surface:

$$
\frac{1}{\beta} \frac{\partial F_{1}^{(0)}}{\partial \beta}+\frac{\partial^{2} F_{1}^{(0)}}{\partial \beta^{2}}=0,-\frac{\partial^{2} F_{1}^{(0)}}{\partial \beta \partial \eta}=0, \lambda_{33}\left(-\frac{\partial \phi_{1}^{(0)}}{\partial \eta}\right)=0, \text { at } \eta=\frac{1}{2} ;
$$

and for the fixed peripheral:

$$
u_{z}=0, u_{r}=0, \frac{\partial u_{z}}{\partial \beta}=0, \text { at } \beta=\frac{a}{h} \text { and } \eta=\eta_{n} .
$$

The integration constants are determined as

$$
\left\{\begin{array}{l}
A_{1}^{(0)}=A_{2}^{(0)}=C_{1}^{(0)}=C_{2}^{(0)}=C_{3}^{(0)}=C_{4}^{(0)}=C_{6}^{(0)}=0, \\
A_{3}^{(0)}=\frac{q}{e^{\alpha}+e^{-\alpha}-\alpha^{2}-2}\left(-\frac{\alpha^{2}}{4}\right) \\
A_{4}^{(0)}=\frac{q}{e^{\alpha}+e^{-\alpha}-\alpha^{2}-2}\left(\frac{-2 e^{-\alpha}+\alpha^{2}+2 \alpha+2}{8}\right), \\
B_{1}^{(0)}=\frac{q}{e^{\alpha}+e^{-\alpha}-\alpha^{2}-2} \frac{\alpha^{2}\left(1+S_{12}\right)\left[(\alpha+2) e^{-\alpha / 2}+(\alpha-2) e^{\alpha / 2}\right]}{128}, \\
B_{2}^{(0)}=\frac{q}{e^{\alpha}+e^{-\alpha}-\alpha^{2}-2} \frac{\alpha^{3}\left(1+S_{12}\right)\left(e^{\alpha / 2}-e^{-\alpha / 2}\right)}{64} .
\end{array}\right.
$$

Note that $C_{5}^{(0)}$ is the constant term of electrical potential function $\phi$, and has no effect on the electrical field (this may be easily seen from Equation (6)); therefore there is no need to determine it. This conclusion is also applicable to the next first-order perturbation. In addition, we also note that unlike Equations (31)-(32), Equation (33) is not expressed in terms of two stress functions. It may be more convenient to use this boundary condition directly expressed in terms of displacement. For example, $A_{5}^{(0)}$ and $A_{6}^{(0)}$ are still not determined, and will be given in Appendix $\mathrm{A}$, in which Equation (33) is used.

\subsection{First-Order Perturbation Solution}

There are three perturbation parameters, $D_{31}, D_{33}$, and $D_{15}$, in our study. Next, we will derive the first-order perturbation equation and corresponding boundary conditions with respect to the three parameters, respectively.

For $D_{31}$, substituting Equation (19) into Equations (16)-(18), we have the first-order perturbation equations with respect to $D_{31}$ as follows:

$$
\begin{gathered}
2 \alpha \beta \frac{\partial^{2} F_{1}^{(0)}}{\partial \eta^{2}}+2 \beta \frac{\partial^{3} F_{1}^{(0)}}{\partial \eta^{3}}+\alpha \frac{\partial \psi_{1}^{(0)}}{\partial \beta}+\alpha \beta \frac{\partial^{2} \psi_{1}^{(0)}}{\partial \beta^{2}}+\frac{\partial^{2} \psi_{1}^{(0)}}{\partial \beta \partial \eta}+\beta \frac{\partial^{3} \psi_{1}^{(0)}}{\partial \beta^{2} \partial \eta}-\frac{\partial \phi_{1}^{(1)}}{\partial \beta} \\
-\alpha \beta \lambda_{33} \frac{\partial \phi_{1}^{(1)}}{\partial \eta}-\beta \frac{\partial^{2} \phi_{1}^{(1)}}{\partial \beta^{2}}-\beta \lambda_{33} \frac{\partial^{2} \phi_{1}^{(1)}}{\partial \eta^{2}}=0 \\
\left(\alpha^{2} \beta^{2} S_{13}+2 S_{33}\right) \frac{\partial F_{1}^{(1)}}{\partial \beta}+\left(\alpha^{2} \beta^{3} S_{13}-2 \beta S_{33}\right) \frac{\partial^{2} F_{1}^{(1)}}{\partial \beta^{2}}+2 \alpha \beta^{2} S_{13} \frac{\partial^{2} F_{1}^{(1)}}{\partial \beta \partial \eta}+\alpha^{2} \beta^{3}\left(1+S_{12}\right) \\
\frac{\partial^{2} F_{1}^{(1)}}{\partial \eta^{2}}+\beta^{2} S_{33} \frac{\partial^{3} F_{1}^{(1)}}{\partial \beta^{3}}+\alpha \beta^{3}\left(2 S_{13}+S_{44}\right) \frac{\partial^{3} F_{1}^{(1)}}{\partial \beta^{2} \partial \eta}+\beta^{2} S_{13} \frac{\partial^{3} F_{1}^{(1)}}{\partial \beta \partial \eta^{2}}+2 \alpha \beta^{3}\left(1+S_{12}\right) \frac{\partial^{3} F_{1}^{(1)}}{\partial \eta^{3}} \\
+\beta^{3}\left(3 S_{13}+S_{44}\right) \frac{\partial^{4} F_{1}^{(1)}}{\partial \beta^{2} \partial \eta^{2}}+\beta^{3} S_{33} \frac{\partial^{4} F_{1}^{(1)}}{\partial \beta^{4}}+\beta^{3}\left(1+S_{12}\right) \frac{\partial^{4} F_{1}^{(1)}}{\partial \eta^{4}}+\left(\alpha^{2} \beta^{3} S_{12}-2 \beta S_{13}\right) \frac{\partial^{2} \psi_{1}^{(1)}}{\partial \beta^{2}} \\
+\left(\alpha^{2} \beta^{2}+2 S_{13}\right) \frac{\partial \psi_{1}^{(1)}}{\partial \beta}+2 \alpha \beta^{2} \frac{\partial^{2} \psi_{1}^{(1)}}{\partial \beta \partial \eta}+2 \alpha \beta^{3} S_{12} \frac{\partial^{3} \psi_{1}^{(1)}}{\partial \beta^{2} \partial \eta}+\beta^{2} S_{13} \frac{\partial^{3} \psi_{1}^{(1)}}{\partial \beta^{3}}+\beta^{2} \frac{\partial^{3} \psi_{1}^{(1)}}{\partial \beta \partial \eta^{2}} \\
+\beta^{3} S_{13} \frac{\partial^{4} \psi_{1}^{(1)}}{\partial \beta^{4}}+\beta^{3} S_{12} \frac{\partial^{4} \psi_{1}^{(1)}}{\partial \beta^{2} \partial \eta^{2}}-\alpha^{2} \beta^{3} \frac{\partial \phi_{1}^{(0)}}{\partial \eta}-2 \alpha \beta^{3} \frac{\partial^{2} \phi_{1}^{(0)}}{\partial \eta^{2}}-\beta^{3} \frac{\partial^{3} \phi_{1}^{(0)}}{\partial \eta^{3}}=0
\end{gathered}
$$

and

$$
\begin{aligned}
& -S_{13} \frac{\partial F_{1}^{(1)}}{\partial \beta}+\beta S_{13} \frac{\partial^{2} F_{1}^{(1)}}{\partial \beta^{2}}+\beta^{2} S_{13} \frac{\partial^{3} F_{1}^{(1)}}{\partial \beta^{3}}+\beta^{2}\left(1+S_{12}\right) \frac{\partial^{3} F_{1}^{(1)}}{\partial \beta \partial \eta^{2}}-\frac{\partial \psi_{1}^{(1)}}{\partial \beta}+\beta \frac{\partial^{2} \psi_{1}^{(1)}}{\partial \beta^{2}} \\
& +\beta^{2} \frac{\partial^{3} \psi_{1}^{(1)}}{\partial \beta^{3}}-\beta^{2} \frac{\partial^{2} \phi_{1}^{(0)}}{\partial \beta \partial \eta}=0
\end{aligned}
$$


which may be solved under the following boundary conditions: for the upper and lower surfaces, we have

$$
\left\{\begin{array}{l}
\frac{1}{\beta} \frac{\partial F_{1}^{(1)}}{\partial \beta}+\frac{\partial^{2} F_{1}^{(1)}}{\partial \beta^{2}}=0,-\frac{\partial^{2} F_{1}^{(1)}}{\partial \beta \partial \eta}=0, \\
2 \frac{\partial^{2} F_{1}^{(0)}}{\partial \eta^{2}}+\frac{1}{\beta} \frac{\partial \psi_{1}^{(0)}}{\partial \beta}+\frac{\partial^{2} \psi_{1}^{(0)}}{\partial \beta^{2}}-\lambda_{33} \frac{\partial \phi_{1}^{(1)}}{\partial \eta}=0,
\end{array} \text { at } \eta= \pm \frac{1}{2}\right.
$$

and for the fixed peripheral,

$$
\begin{aligned}
& S_{12}\left(\frac{\partial^{2} F_{1}^{(1)}}{\partial \eta^{2}}+\frac{1}{\beta} \frac{\partial \psi_{1}^{(1)}}{\partial \beta}\right)+\left(\frac{\partial^{2} F_{1}^{(1)}}{\partial \eta^{2}}+\frac{\partial^{2} \psi_{1}^{(1)}}{\partial \beta^{2}}\right) \\
& +S_{13}\left(\frac{1}{\beta} \frac{\partial F_{1}^{(1)}}{\partial \beta}+\frac{\partial^{2} F_{1}^{(1)}}{\partial \beta^{2}}\right)-\frac{\partial \phi_{1}^{(0)}}{\partial \eta}=0, \text { at } \beta=\frac{a}{h}
\end{aligned}
$$

The detailed derivations for unknown functions, $F_{1}^{(1)}, \psi_{1}^{(1)}$, and $\phi_{1}^{(1)}$ are given in Appendix B.

Similarly, we may have the first-order perturbation equations with respect to $D_{33}$ :

$$
\begin{gathered}
\alpha \frac{\partial F_{1}^{(0)}}{\partial \beta}+\alpha \beta \frac{\partial^{2} F_{1}^{(0)}}{\partial \beta^{2}}+\frac{\partial^{2} F_{1}^{(0)}}{\partial \beta \partial \eta}+\beta \frac{\partial^{3} F_{1}^{(0)}}{\partial \beta^{2} \partial \eta} \\
-\frac{\partial \phi_{2}^{(1)}}{\partial \beta}-\alpha \beta \lambda_{33} \frac{\partial \phi_{2}^{(1)}}{\partial \eta}-\beta \frac{\partial^{2} \phi_{2}^{(1)}}{\partial \beta^{2}}-\beta \lambda_{33} \frac{\partial^{2} \phi_{2}^{(1)}}{\partial \eta^{2}}=0 \\
\left(\alpha^{2} \beta^{2} S_{13}+2 S_{33}\right) \frac{\partial F_{2}^{(1)}}{\partial \beta}+\left(\alpha^{2} \beta^{3} S_{13}-2 \beta S_{33}\right) \frac{\partial^{2} F_{2}^{(1)}}{\partial \beta^{2}}+2 \alpha \beta^{2} S_{13} \frac{\partial^{2} F_{2}^{(1)}}{\partial \beta \partial \eta}+\beta^{2} S_{33} \frac{\partial^{3} F_{2}^{(1)}}{\partial \beta^{3}} \\
+\alpha^{2} \beta^{3}\left(S_{11}+S_{12}\right) \frac{\partial^{2} F_{2}^{(1)}}{\partial \eta^{2}}+\alpha \beta^{3}\left(2 S_{13}+S_{44}\right) \frac{\partial^{3} F_{2}^{(1)}}{\partial \beta^{2} \partial \eta}+\beta^{2} S_{13} \frac{\partial^{3} F_{2}^{(1)}}{\partial \beta \partial \eta^{2}}+\beta^{3}\left(S_{11}+S_{12}\right) \frac{\partial^{4} F_{2}^{(1)}}{\partial \eta^{4}} \\
+2 \alpha \beta^{3}\left(S_{11}+S_{12}\right) \frac{\partial^{3} F_{2}^{(1)}}{\partial \eta^{3}}+\beta^{3} S_{33} \frac{\partial^{4} F_{2}^{(1)}}{\partial \beta^{4}}+\beta^{3}\left(3 S_{13}+S_{44}\right) \frac{\partial^{4} F_{2}^{(1)}}{\partial \beta^{2} \partial \eta^{2}}+\left(\alpha^{2} \beta^{2} S_{11}+2 S_{13}\right) \frac{\partial \psi_{2}^{(1)}}{\partial \beta} \\
+2 \alpha \beta^{2} S_{11} \frac{\partial^{2} \psi_{2}^{(1)}}{\partial \beta \partial \eta}+\left(\alpha^{2} \beta^{3} S_{12}-2 \beta S_{13}\right) \frac{\partial^{2} \psi_{2}^{(1)}}{\partial \beta^{2}}+\beta^{2} S_{13} \frac{\partial^{3} \psi_{2}^{(1)}}{\partial \beta^{3}}+\beta^{2} S_{11} \frac{\partial^{3} \psi_{2}^{(1)}}{\partial \beta \partial \eta^{2}}+\beta^{3} S_{13} \frac{\partial^{4} \psi_{2}^{(1)}}{\partial \beta^{4}} \\
+2 \alpha \beta^{3} S_{12} \frac{\partial^{3} \psi_{2}^{(1)}}{\partial \beta^{2} \partial \eta}+\beta^{3} S_{12} \frac{\partial^{4} \psi_{2}^{(1)}}{\partial \beta^{2} \partial \eta^{2}}-\beta^{3} \frac{\partial^{3} \phi_{1}^{(0)}}{\partial \beta^{2} \partial \eta}=0
\end{gathered}
$$

and

$$
\begin{aligned}
& -S_{13} \frac{\partial F_{2}^{(1)}}{\partial \beta}+\beta S_{13} \frac{\partial^{2} F_{2}^{(1)}}{\partial \beta^{2}}+\beta^{2}\left(1+S_{12}\right) \frac{\partial^{3} F_{2}^{(1)}}{\partial \beta \partial \eta^{2}}+\beta^{2} S_{13} \frac{\partial^{3} F_{2}^{(1)}}{\partial \beta^{3}} \\
& -\frac{\partial \psi_{2}^{(1)}}{\partial \beta}+\beta \frac{\partial^{2} \psi_{2}^{(1)}}{\partial \beta^{2}}+\beta^{2} \frac{\partial^{3} \psi_{2}^{(1)}}{\partial \beta^{3}}=0
\end{aligned}
$$

which may be solved under the following boundary conditions: for the upper and lower surfaces, we have

$$
\left\{\begin{array}{l}
\frac{1}{\beta} \frac{\partial F_{2}^{(1)}}{\partial \beta}+\frac{\partial^{2} F_{2}^{(1)}}{\partial \beta^{2}}=0,-\frac{\partial^{2} F_{2}^{(1)}}{\partial \beta \partial \eta}=0, \\
\frac{1}{\beta} \frac{\partial F_{1}^{(0)}}{\partial \beta}+\frac{\partial^{2} F_{1}^{(0)}}{\partial \beta^{2}}-\lambda_{33} \frac{\partial \phi_{2}^{(1)}}{\partial \eta}=0
\end{array} \text { at } \eta= \pm \frac{1}{2}\right.
$$

and for the fixed peripheral,

$$
S_{12}\left(\frac{\partial^{2} F_{2}^{(1)}}{\partial \eta^{2}}+\frac{1}{\beta} \frac{\partial \psi_{2}^{(1)}}{\partial \beta}\right)+\left(\frac{\partial^{2} F_{2}^{(1)}}{\partial \eta^{2}}+\frac{\partial^{2} \psi_{2}^{(1)}}{\partial \beta^{2}}\right)+S_{13}\left(\frac{1}{\beta} \frac{\partial F_{2}^{(1)}}{\partial \beta}+\frac{\partial^{2} F_{2}^{(1)}}{\partial \beta^{2}}\right)=0, \text { at } \beta=\frac{a}{h} .
$$

The detailed derivations for unknown functions, $F_{2}^{(1)}, \psi_{2}^{(1)}$ and $\phi_{2}^{(1)}$, are given in Appendix B.

We similarly may have the first-order perturbation equations with respect to $D_{15}$ :

$$
\frac{\partial^{2} F_{1}^{(0)}}{\partial \beta \partial \eta}+\beta \frac{\partial^{3} F_{1}^{(0)}}{\partial \beta^{2} \partial \eta}+\frac{\partial \phi_{3}^{(1)}}{\partial \beta}+\alpha \beta \lambda_{33} \frac{\partial \phi_{3}^{(1)}}{\partial \eta}+\beta \frac{\partial^{2} \phi_{3}^{(1)}}{\partial \beta^{2}}+\beta \lambda_{33} \frac{\partial^{2} \phi_{3}^{(1)}}{\partial \eta^{2}}=0,
$$




$$
\begin{aligned}
& \left(\alpha^{2} \beta^{2} S_{13}+2 S_{33}\right) \frac{\partial F_{3}^{(1)}}{\partial \beta}+\left(\alpha^{2} \beta^{3} S_{13}-2 \beta S_{33}\right) \frac{\partial^{2} F_{3}^{(1)}}{\partial \beta^{2}}+2 \alpha \beta^{2} S_{13} \frac{\partial^{2} F_{3}^{(1)}}{\partial \beta \partial \eta} \\
& +\alpha^{2} \beta^{3}\left(S_{11}+S_{12}\right) \frac{\partial^{2} F_{3}^{(1)}}{\partial \eta^{2}}+\beta^{2} S_{33} \frac{\partial^{3} F_{3}^{(1)}}{\partial \beta^{3}}+\alpha \beta^{3}\left(2 S_{13}+S_{44}\right) \frac{\partial^{3} F_{3}^{(1)}}{\partial \beta^{2} \partial \eta} \\
& +\beta^{2} S_{13} \frac{\partial^{3} F_{3}^{(1)}}{\partial \beta \partial \eta^{2}}+2 \alpha \beta^{3}\left(S_{11}+S_{12}\right) \frac{\partial^{3} F_{3}^{(1)}}{\partial \eta^{3}}+\beta^{3} S_{33} \frac{\partial^{4} F_{3}^{(1)}}{\partial \beta^{4}}+\beta^{3}\left(3 S_{13}+S_{44}\right) \\
& \frac{\partial^{4} F_{3}^{(1)}}{\partial \beta^{2} \partial \eta^{2}}+\beta^{3}\left(S_{11}+S_{12}\right) \frac{\partial^{4} F_{3}^{(1)}}{\partial \eta^{4}}+\left(\alpha^{2} \beta^{2} S_{11}+2 S_{13}\right) \frac{\partial \psi_{3}^{(1)}}{\partial \beta}+\left(\alpha^{2} \beta^{3} S_{12}-2 \beta S_{13}\right) \\
& \frac{\partial^{2} \psi_{3}^{(1)}}{\partial \beta^{2}}+2 \alpha \beta^{2} S_{11} \frac{\partial^{2} \psi_{3}^{(1)}}{\partial \beta \partial \eta}+\beta^{2} S_{13} \frac{\partial^{3} \psi_{3}^{(1)}}{\partial \beta^{3}}+2 \alpha \beta^{3} S_{12} \frac{\partial^{3} \psi_{3}^{(1)}}{\partial \beta^{2} \partial \eta}+\beta^{2} S_{11} \frac{\partial^{3} \psi_{3}^{(1)}}{\partial \beta \partial \eta^{2}} \\
& +\beta^{3} S_{13} \frac{\partial^{4} \psi_{3}^{(1)}}{\partial \beta^{4}}+\beta^{3} S_{12} \frac{\partial^{4} \psi_{3}^{(1)}}{\partial \beta^{2} \partial \eta^{2}}+\alpha \beta^{3} \frac{\partial^{2} \phi_{1}^{(0)}}{\partial \beta^{2}}+\beta^{3} \frac{\partial^{3} \phi_{1}^{(0)}}{\partial \beta^{2} \partial \eta}=0
\end{aligned}
$$

and

$$
\begin{aligned}
& -S_{13} \frac{\partial F_{3}^{(1)}}{\partial \beta}+\beta S_{13} \frac{\partial^{2} F_{3}^{(1)}}{\partial \beta^{2}}+\beta^{2}\left(1+S_{12}\right) \frac{\partial^{3} F_{3}^{(1)}}{\partial \beta \partial \eta^{2}}+\beta^{2} S_{13} \frac{\partial^{3} F_{3}^{(1)}}{\partial \beta^{3}} \\
& -\frac{\partial \psi_{3}^{(1)}}{\partial \beta}+\beta \frac{\partial^{2} \psi_{3}^{(1)}}{\partial \beta^{2}}+\beta^{2} \frac{\partial^{3} \psi_{3}^{(1)}}{\partial \beta^{3}}=0
\end{aligned}
$$

which may be solved under the following boundary conditions: for the upper and lower surfaces, we have

$$
\left\{\begin{array}{l}
\frac{1}{\beta} \frac{\partial F_{3}^{(1)}}{\partial \beta}+\frac{\partial^{2} F_{3}^{(1)}}{\partial \beta^{2}}=0,-\frac{\partial^{2} F_{3}^{(1)}}{\partial \beta \partial \eta}=0, \\
-\lambda_{33} \frac{\partial \phi_{3}^{(1)}}{\partial \eta}=0,
\end{array} \text { at } \eta= \pm \frac{1}{2}\right.
$$

and for the fixed peripheral,

$$
S_{12}\left(\frac{\partial^{2} F_{3}^{(1)}}{\partial \eta^{2}}+\frac{1}{\beta} \frac{\partial \psi_{3}^{(1)}}{\partial \beta}\right)+\left(\frac{\partial^{2} F_{3}^{(1)}}{\partial \eta^{2}}+\frac{\partial^{2} \psi_{3}^{(1)}}{\partial \beta^{2}}\right)+S_{13}\left(\frac{1}{\beta} \frac{\partial F_{3}^{(1)}}{\partial \beta}+\frac{\partial^{2} F_{3}^{(1)}}{\partial \beta^{2}}\right)=0, \text { at } \beta=\frac{a}{h}
$$

The detailed derivations for unknown functions, $F_{3}^{(1)}, \psi_{3}^{(1)}$, and $\phi_{3}^{(1)}$, are given in Appendix B.

Suppose we stop here for the complexity of the derivation process. Some basic conclusions may be drawn from the zero-order and first-order perturbation solutions presented. First, we note that the undetermined integral constants, $A^{(i)}, B^{(i)}$, and $C^{(i)}(i=0,1)$ in zero-order and first-order perturbation solutions are related to $f_{j 1}^{(i)}(\eta), f_{j 2}^{(i)}(\eta), f_{j 3}^{(i)}(\eta), g_{j 1}^{(i)}(\eta), g_{j 2}^{(i)}(\eta), h_{j 1}^{(i)}(\eta), h_{j 2}^{(i)}(\eta)$, and $h_{j 3}^{(i)}(\eta)$, thus associating with $F_{j}^{(i)}(\beta, \eta), \psi_{j}^{(i)}(\beta, \eta)$, and $\phi_{j}^{(i)}(\beta, \eta)$. More specifically, in the zero-order solution, $C^{(0)}=0, A^{(0)} \neq 0$, and $B^{(0)} \neq 0$ will lead to the stress functions $F_{j}^{(0)}(\beta, \eta)$ and $\psi_{j}^{(0)}(\beta, \eta)$, which are not zero while the potential function $\psi_{j}^{(0)}(\beta, \eta)$ is zero. Therefore, it can be concluded that the zero-order solution actually corresponds to the mechanical solution. At the same time, in the first-order solution, $A^{(1)}=0, B^{(1)}=0$, and $C^{(1)} \neq 0$ will lead to the potential function $\phi_{j}^{(1)}(\beta, \eta)$, which is not zero while the stress functions $F_{j}^{(1)}(\beta, \eta)$ and $\psi_{j}^{(1)}(\beta, \eta)$ are zero; therefore, it can be concluded that the first-order solution actually corresponds to the electrical solution. Similar conclusions may be found in our previous study on beams [41].

For elastic stress and elastic displacement, in a purely piezoelectric problem (without functionally-graded characteristics), we should note that some solutions of the elastic stress are the same as those of classical problems (without piezoelectric effect and without functionally-graded characteristics), since the introduction of the piezoelectric constitutive relationship will not change the final stress result. On the contrary, this relationship will change the final displacement result. Therefore, we may compare the stress solution from the classical problem with our results obtained here-for example, the expressions for $\sigma_{z}$ and $\tau_{r z}$ in classical problems [44] (also in a purely piezoelectric problem) are 


$$
\left\{\begin{array}{l}
\frac{\sigma_{z}}{q}=-2 \frac{z^{3}}{h^{3}}+\frac{3}{2} \frac{z}{h}-\frac{1}{2} \\
\frac{\tau_{r z}}{q}=3 \frac{r}{h}\left(\frac{z^{2}}{h^{2}}-\frac{1}{4}\right)
\end{array},\right.
$$

where $\sigma_{z}, \tau_{r z}$, and $q$ are dimensional quantities. Specially, if we let $\alpha=0$ (or $\alpha \rightarrow 0$ ) in the solution derived in this study, the obtained solution should be the solution of a purely piezoelectric thin circular plate without functionally-graded characteristics, as shown in Equation (50). For this purpose, we compute $\lim _{\alpha \rightarrow 0} \sigma_{z}$ and $\lim _{\alpha \rightarrow 0} \tau_{r z}$, and after noting that $\beta=r / h$ and $\eta=z / h$, the same expressions as Equation (50) are obtained. This verifies the correctness of the perturbation solution from the side.

\section{Numerical Simulation and Comparison with Perturbation Solution}

In order to further verify the correctness of the multi-parameter perturbation solution, a FGPM thin circular plate subjected to uniformly-distributed loads was calculated via a numerical analysis program, thus allowing corresponding comparison with the analytical expression obtained above.

\subsection{Numerical Simulation}

ABAQUS software is one of the large-scale finite element software programs at present that can analyze complex mechanical problems in engineering, including the problem of piezoelectric materials. However, the software itself does not include functionally-graded properties of materials varying in a form of continuous function. For this purpose, we resorted to layer-wise theory to simulate the problem studied.

Another problem, which should be noted here, is different types of constitutive models of the piezoelectrical materials. In ABAQUS, an $e$-type constitutive equation for piezoelectrical materials is adopted, such that

$$
\left\{\begin{array}{l}
\sigma_{i j}=D_{i j k l}^{E} \varepsilon_{k l}-e_{m i j}^{\varphi} E_{m} \\
q_{i j}=e_{m i j}^{\varphi} \varepsilon_{j k}+D_{i j}^{\varphi(\varepsilon)} E_{j}
\end{array}(i, j, k, l, m=1,2,3),\right.
$$

where $\sigma_{i j}$ is the stress component, $\varepsilon_{i j}$ is the strain component, $q_{i j}$ is the electrical displacement component, $D_{i j k l}^{E}$ is the stiffness coefficient matrix, $D_{i j}^{\varphi(\varepsilon)}$ is the piezoelectrical stress constants matrix, $e_{m i j}^{\varphi}$ is the dielectric constant matrix, and $E_{m}$ and $E_{j}$ are electrical field strengths. We note that in the theoretical derivation presented above and the properties of materials given below, the $d$-type constitutive equation of piezoelectric materials was adopted. Therefore, the transformation of these material parameters in different types of the constitutive model was needed. Considering the length limit of the paper, however, we will not repeat it here. More details may be found in our previous study [45].

The other detailed steps for modeling and simulation are as follows:

(i) Establishment of entity structure

The solid model of an FGPM thin circular plate is established, in which the thickness of the plate $h=20 \mathrm{~mm}$ and the radius of the plate $a=300 \mathrm{~mm}$.

\section{(ii) Layering and determination of materials properties in each layer}

According to conventional practice, we still adopted layer-wise theory to simulate the functionally-graded properties varying in the thickness direction. Without losing computational accuracy, the plate was divided into a moderate number of layers; the physical parameters of the material in each layer was regarded as the same, thus indirectly realizing the continuous variation of material properties throughout the thickness direction. The change in material properties in each layer will follow the form that has been adopted in our theoretical derivation,

$$
s_{i j}=s_{i j}^{0} \cdot e^{2 \eta}, d_{i j}=d_{i j}^{0} \cdot e^{2 \eta}, \lambda_{i j}=\lambda_{i j}^{0} \cdot e^{2 \eta},
$$


where the functionally gradient index is set to $\alpha=2$. The material constant at $\eta=0$ is shown in Table 1 , which may be input into ABAQUS directly.

Table 1. Physical properties of material $\mathrm{PbZrTiO}_{3}-4$ (Generally abbreviated as PZT-4).

\begin{tabular}{cccccccccc}
\hline & \multicolumn{3}{c}{$\begin{array}{c}\text { Elastic Constants } \\
\left(\mathbf{1 0}^{-\mathbf{1 2}} \mathbf{~ m}^{\mathbf{2}} \cdot \mathbf{N}^{-\mathbf{1}} \mathbf{)}\right.\end{array}$} & & \multicolumn{2}{c}{$\begin{array}{c}\text { Piezoelectric Constants } \\
\left(\mathbf{1 0}^{-\mathbf{1 2}} \mathbf{C} \cdot \mathbf{N}^{-\mathbf{1}}\right)\end{array}$} & \multicolumn{2}{c}{$\begin{array}{c}\text { Dielectric Constants } \\
\left(\mathbf{( 1 0}^{-\mathbf{8}} \mathbf{F} \cdot \mathbf{m}^{-\mathbf{1}} \mathbf{)}\right.\end{array}$} \\
\hline$s_{11}^{0}$ & $s_{12}^{0}$ & $s_{13}^{0}$ & $s_{33}^{0}$ & $s_{44}^{0}$ & $d_{31}^{0}$ & $d_{33}^{0}$ & $d_{15}^{0}$ & $\lambda_{11}^{0}$ & $\lambda_{33}^{0}$ \\
12.4 & -4.05 & -5.52 & 16.1 & 39.1 & -135 & 300 & 525 & 1.301 & 1.151 \\
\hline
\end{tabular}

In our real simulation, a plate with thickness $20 \mathrm{~mm}$ is divided into 19 layers along the thickness direction, in which the thickness of the middle layer is $2 \mathrm{~mm}$, the upper and lower layer numbers are both $9 \mathrm{~mm}$, and the thickness of each layer is $1 \mathrm{~mm}$, as shown in Figure 2. Therefore, via the layered model, the physical parameters in each layer may be input into the program layer by layer.

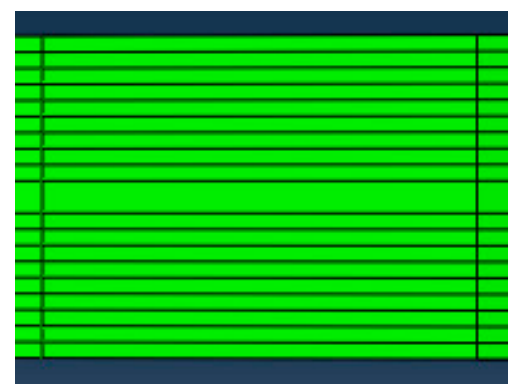

Figure 2. Layered model of an FGPM thin circular plate.

(iii) Establishment of boundary conditions

The boundary condition of the peripheral of the plate was still considered as fully fixed, as shown in Figure 1. For this purpose, we needed to exert constraint at the periphery of the plate, including displacement and rotation.

(iv) Mesh division

In this simulation, an eight-node, linear piezoelectric brick C3D8E was adopted. Considering that the assumed material property varies more uniformly, mesh of the same density was adopted. The global approximate size was set to be 0.03 .

(v) Step module and adding loads

An analysis step called "static general" was established to apply the load. In this simulation, only a uniformly distributed load form was considered. For this purpose, a uniformly distributed load $\left(q=1 \mathrm{~N} / \mathrm{m}^{2}\right)$ along the positive direction of $z$ axis was applied on the upper surface of the plate.

(vi) Operation and results output

After a job was established, the job was submitted for calculation and output of the results.

\subsection{Comparison with Perturbation Solution}

In this section, we will use the results obtained in Section 3 to compute the theoretical solution for some special quantities in question, in which the gradient index $\alpha=2$ and other given data is the same as numerical simulation (for example, the physical properties of $\mathrm{PbZrTiO}_{3}-4$ (Generally abbreviated as PZT-4) in a dimensionless form are given in Table 2, according to Equation (15a)), thus comparing the theoretical solution with the numerical example in a dimensionless form, as shown in 
Figure 3. Note that all stress components in Figure 3 are given in dimensionless values (please refer to Equation (15b)).

Table 2. Dimensionless physical parameters of material PZT-4.

\begin{tabular}{cccccccccc}
\hline & \multicolumn{3}{c}{ Elastic Constants } & \multicolumn{3}{c}{ Piezoelectric Constants } & \multicolumn{2}{c}{ Dielectric Constants } \\
\hline$S_{11}$ & $S_{12}$ & $S_{13}$ & $S_{33}$ & $S_{44}$ & $D_{31}$ & $D_{33}$ & $D_{15}$ & $\lambda_{11}$ & $\lambda_{33}$ \\
1 & -0.327 & -0.445 & 1.298 & 3.153 & -0.336 & 0.747 & 1.307 & 1 & 0.885 \\
\hline
\end{tabular}

Figure 3 a shows the variation of vertical deflection $u_{z}$ at the neutral layer $\eta=\eta_{n}$ with the radial coordination $\beta$. It is easy to see that the theoretical solution and numerical result increase nonlinearly with the distance from the plate edge; in particular, the two deflection values achieve their maximum at the plate's center. The two curves agree well with each other, and the theoretical curve is slightly higher than the numerical one.

Figure $3 \mathrm{~b}$ shows the variation of the radial stress $\sigma_{r}$ at the plate center $\beta=0$, with thickness direction coordinate $\eta$. The two curves basically agree, and there are positive and negative radial stresses in them. The location at which the radial stress is zero is exactly the location of the neutral layer. Obviously, the neutral layer is not at $\eta=0$. The greater the distance from the neutral layer, the greater the radial stress. This is similar to the distribution of radial stress in general bending problems.

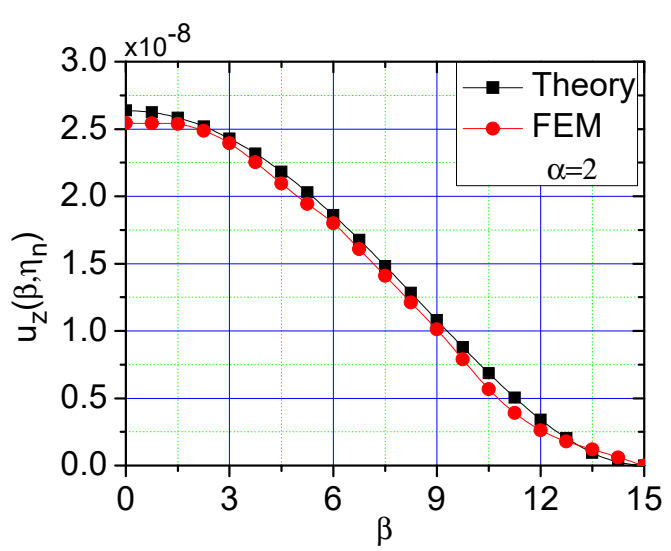

(a)

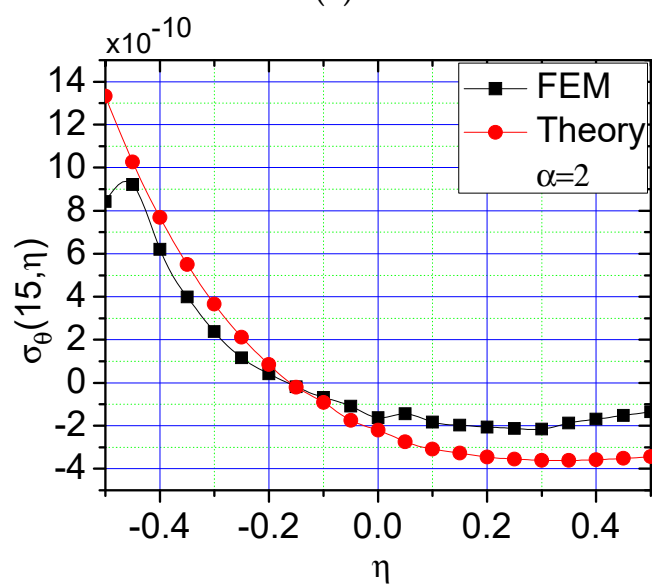

(c)

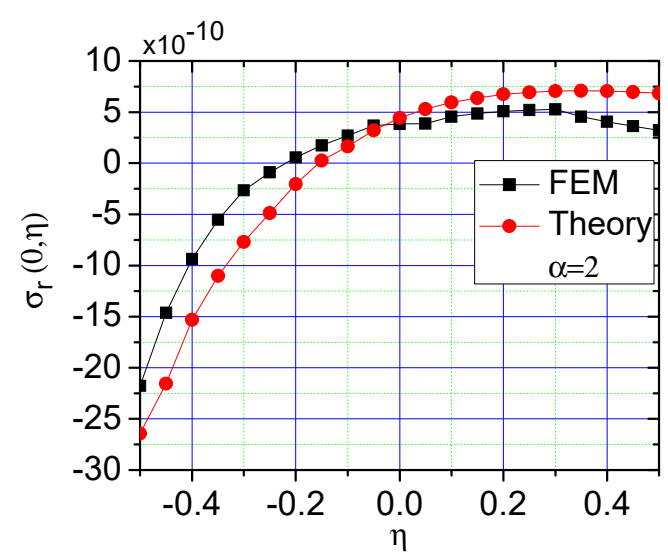

(b)

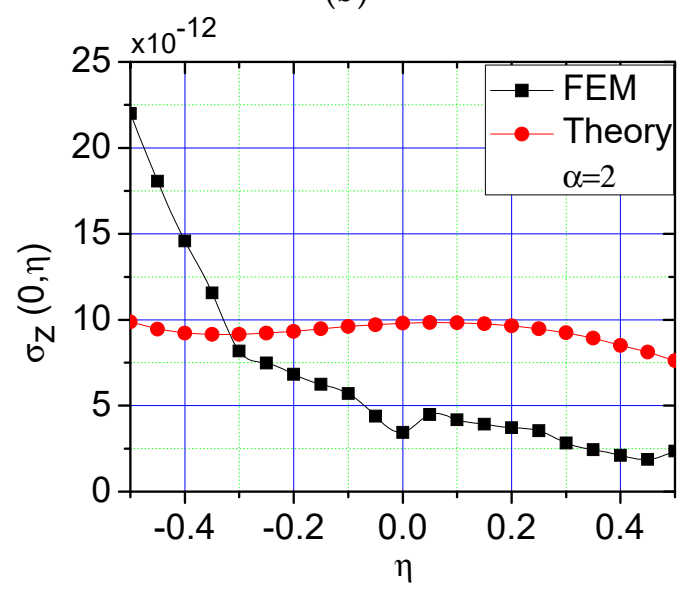

(d)

Figure 3. Comparisons between theoretical solutions and numerical results: (a) deflection at the neutral layer, (b) radial stress at plate center, (c) circumferential stress at plate's edge, and (d) z-direction stress at plate center. 
Figure $3 c$ gives the variation of circumferential stress $\sigma_{\theta}$ at the plate edge $\beta=15$ with thickness direction coordinate $\eta$. The distribution of the two curves is basically consistent, and the maximum value of the circumferential stress occurs at the upper surface.

Figure $3 \mathrm{~d}$ gives the variation of $z$-direction stressor $\sigma_{z}$ at the plate center, multiplied by $s_{11}^{0}=12.4 \times 10^{-12} \mathrm{~m}^{2} \mathrm{~N}^{-1}$ due to the need for normalization, with thickness direction coordinate $\eta$. The $z$-direction stress at the upper surface of the theoretical curve gives about $\sigma_{z}=10 \times 10^{-12}$, while the real boundary condition is $\sigma_{z}=12.4 \times 10^{-12}$ (note that $q=1 \mathrm{~N} / \mathrm{m}^{2}$; thus, the dimensionless value is $\left.12.4 \times 10^{-12} \mathrm{~m}^{2} \mathrm{~N}^{-1} \times 1 \mathrm{~N} / \mathrm{m}^{2}=12.4 \times 10^{-12}\right)$. The two curves have the same variation tendency; however, there is still some difference between them. It is easy to see that in the area near the upper surface, or at the load action surface, the numerical results are quite different from the theoretical values, which is mainly due to the large discreteness of the finite element calculation results near the load action surface. Fortunately, z-direction stress in thin plates is a relatively secondary stress, like the extrusion stress along the vertical direction in the beam. Compared with the radial stress and circumferential stress, the value of $z$-direction stress is negligibly small; this may be seen by observing the values of the coordinate axis from Figure $3 b-d$.

In addition, Table 3 gives the maximum values of vertical deflections, radial and circumferential stresses, and the relative errors between theoretical solutions and numerical results. It can be seen from Table 3 that the error of deflection is very small, and the error of the maximum circumferential stress is relatively large, which is also related to the large discreteness of the numerical results of the finite element method on the upper surface on which the load acts.

Table 3. The maximum values and relative errors.

\begin{tabular}{cccc}
\hline & $\boldsymbol{u}_{\boldsymbol{z}, \max }\left(\times 10-{ }^{8}\right)^{1}$ & $\sigma_{r, \max }\left(\times 10-{ }^{10}\right)^{2}$ & $\sigma_{\theta, \max }\left(\times 10-{ }^{10}\right)^{3}$ \\
\hline Theoretical solutions & 2.637 & -26.082 & 13.415 \\
Numerical results & 2.599 & -22.143 & 9.582 \\
Relative errors & $1.44 \%$ & $15.10 \%$ & $28.57 \%$ \\
\hline 1,2 and $^{3}$ denote the maximum values of axial displacement, radial stresses and circumferential stresses, respectively.
\end{tabular}

\section{Results and Discussions}

After the validity of the multi-parameter perturbation solution is verified by numerical simulation, the perturbation solution may be used to discuss some interesting topics, including the effect of the functional gradient index on the solution, as well as the deflection difference between FGPM plates and FGM plates.

\subsection{Effect of Gradient Index on Solution}

To analyze the effect of the gradient index on the solution, we select some values of gradient index, i.e., $\alpha=-2,1,0.01,2$, and 5, in which $\alpha=0.01$ may approximately stand for the case that the gradient index is zero, since $\alpha$ cannot be set to zero in our numerical computation. For this purpose, the variations of mechanical stresses and displacements, as well as electrical potentials, electrical field intensities, and electrical displacements along the direction of radius or thickness, are plotted in different gradient indices, as shown in Figure 4.

From Figure $4 \mathrm{a}-\mathrm{c}$, it is easy to see that, the curves of $\alpha=2$ and $\alpha=-2$ have certain symmetry. Fhis symmetry is especially obvious in Figure $4 c$, in which the corresponding distributions are mirror images about the line $\eta=0$. This is because the functional gradient function, which we define during our theoretical derivation, is in the form of an exponential function, as shown in Equation (1) or Equation (52). Besides, in Figure $4 \mathrm{a}, \mathrm{b}$, the zero values of $\sigma_{r}$ and $\sigma_{\theta}$ both correspond to the location of the neutral layer. $\tau_{r z}$ is zero at the upper and lower surfaces, and achieves the greatest value at the neutral layer, which is consistent with classical problems. 
Figure $4 \mathrm{~d}$ shows the variation of the deflection $u_{z}$ at the neutral layer along the direction of radius. It is easy to see that under various gradient indices, the maximum deflection is also different. Obviously, due to the difference introduced by the physical parameters of functionally-graded material, the bending stiffness of the plate is different for various gradient indices.

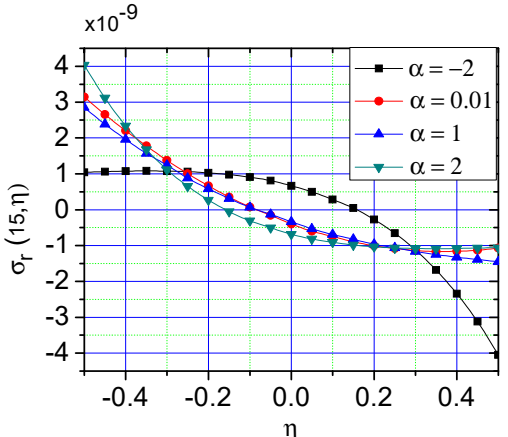

(a)

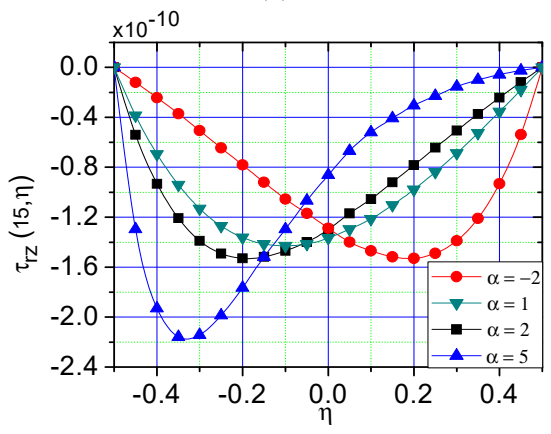

(c)

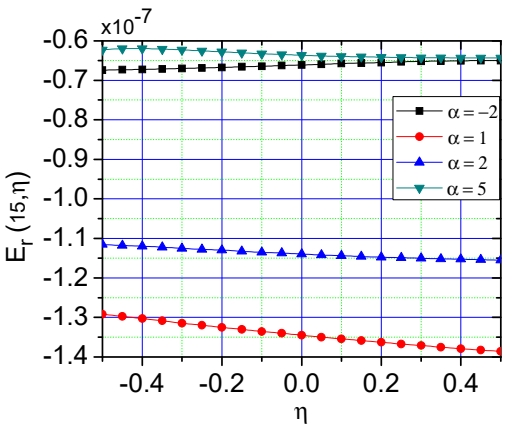

(e)

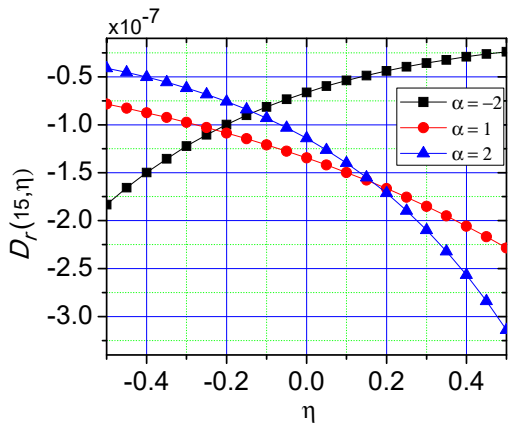

(g)

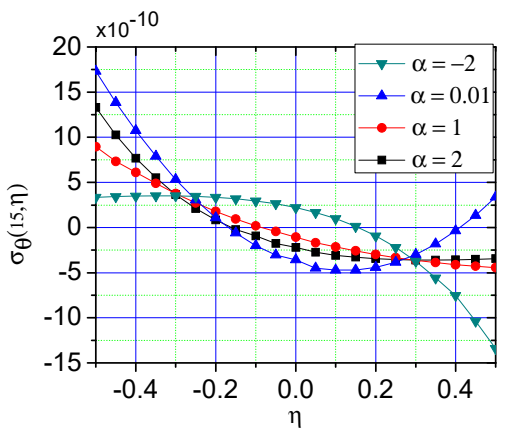

(b)

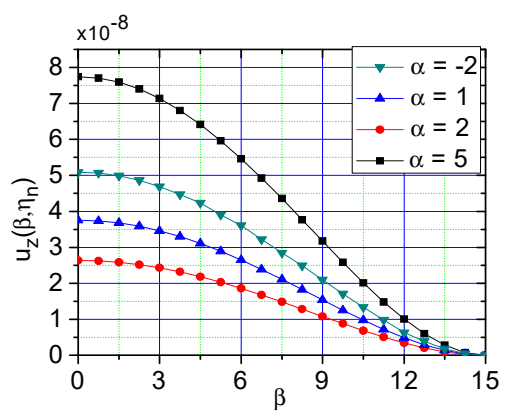

(d)

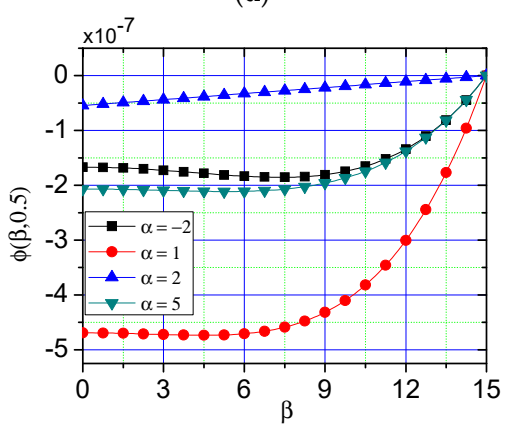

(f)

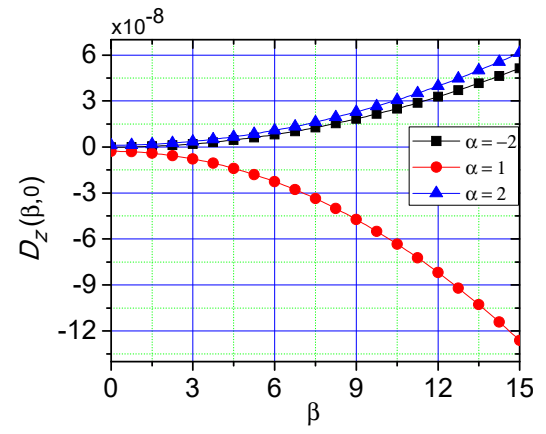

(h)

Figure 4. Variations under various gradient indices: (a) radial stress at plate edge, (b) circumferential stress at the plate's edge, (c) shear stress at plate edge, (d) deflection at the neutral layer, (e) radial field intensity at the plate's edge, (f) electrical potential at the lower surface, (g) radial electrical displacement at the plate's edge, and (h) z-direction electrical displacement at middle layer. 
From Figure 4e, it can be seen that the distribution of $E_{r}$ at the plate's edge under various gradient indices, along the direction of thickness, is basically similar, only with a slight change along the thickness direction. Figure $4 \mathrm{f}$ shows the distribution of $\phi$ at the lower surface along the direction of the radius. Due to the connection to a ground, the electrical potential is zero at the plate's edge $(\beta=15)$ and increases with the distance from the plate edge to the plate center, finally achieving the largest value at the plate center $(\beta=0)$, with the exception of the case where $\alpha=-2$, in which the maximum value is obtained at about $\beta=7.5$.

Figure $4 \mathrm{~g}$ shows that the radial electrical displacement at the plate edge varies with the vertical coordinate. $D_{r}$ changes monotonously along the direction of thickness. When the gradient index is positive or negative, the tendency for change is contrary. Figure $4 \mathrm{~h}$ shows the variation of the $z$-direction electrical displacement at the middle layer with the radial coordinate. It can be seen that under various functional gradient indices, $D_{z}$ at the plate center is zero, and increases gradually from the center to the edge of the plate, achieving its maximum value at the edge. We note that although $D_{r}$ and $D_{z}$ are attributable to electric displacement components, $D_{r}$ and $D_{z}$ may be easily found, as far as their magnitude is concerned. This fact may be used for the simplification in some cases.

\subsection{Deflection Comparison Between FGPM and FGM Plates}

From the multi-parameter perturbation solution for the three piezoelectric coefficients $D_{31}, D_{33}$, and $D_{15}$, we may easily obtain the solution of purely functionally-graded thin plates without the piezoelectric effect, by only letting the three parameters be zero-or, alternatively, by only taking the zero-order perturbation solution in Equation (19). Thus, we have

$$
\left\{\begin{array}{l}
F(\beta, \eta)=F_{1}^{(0)} \\
\psi(\beta, \eta)=\psi_{1}^{(0)} \\
\phi(\beta, \eta)=\phi_{1}^{(0)}
\end{array} .\right.
$$

Due to the fact that the perturbation solution is, in this form, an expansion of the power series with respect to the perturbation parameter, the unperturbed solution (zero-order perturbation solution) may be approximately regarded as a linear part to the solution of the perturbation system (first-order up to the higher-order perturbation solution). Here we focus our discussion on deflection comparisons between FGPM plates and FGM plates; thus, locations of the neutral layer and central deflections of FGPM plates and FGM plates, under various functionally gradient indices, are computed and listed in Table 4.

Table 4. Center deflections $u_{z, \max }\left(\times 10^{-7}\right)$ of FGPM plates and functionally-graded material (FGM) plates.

\begin{tabular}{ccccc}
\hline & $\alpha=-2$ & $\alpha=1$ & $\alpha=2$ & $\alpha=5$ \\
\hline$\eta_{n}$ & 0.157 & -0.082 & -0.157 & -0.307 \\
FGPM & 0.509 & 0.375 & 0.264 & 0.774 \\
FGM & 1.081 & 1.060 & 1.081 & 1.092 \\
\hline
\end{tabular}

It is easy to see that under various gradient indices, the locations for the unknown neutral layer are also different: the greater the absolute value of the gradient index, the greater the distance from the geometric center layer of the plate. In particular, when the absolute values of the gradient indices are equal ( $\alpha=-2$ and $\alpha=2$, for example), the position of the neutral layer is symmetrical about the geometric middle layer. This conclusion has been obtained from our above discussion on Figure $4 \mathrm{a}-\mathrm{c}$. Due to the fact that, under the influence of functionally-graded materials, the neutral layer no longer coincides with the geometric middle plane, the position of the neutral layer is generally close to the layer with a large stiffness coefficient. In addition, it is easy to see that under various gradient indices, there is no more difference in central deflection values for FGM plates, while for FGPM plates, the change of the central deflection is relatively large. 
For further analysis, we may let the deflection at the neutral layer, $u_{z}$, be the following form, according to our obtained solution:

$$
u_{z}=a+b \beta^{2}+c \beta^{4}
$$

where $a, b$, and $c$ are constants, and their values under various gradient indices are listed in Table 5. It is obvious that the constant $a$ corresponds exactly to the central deflection values in Table 4 , which is easily seen from Equation (54).

Table 5. Coefficients of deflection for FGPM plates and FGM plates.

\begin{tabular}{ccccccc}
\hline & \multicolumn{3}{c}{ FGPM Plates } & \multicolumn{3}{c}{ FGM Plates } \\
\cline { 2 - 7 } & $\boldsymbol{a}\left(\times \mathbf{1 0}^{-\mathbf{7}}\right)$ & $\boldsymbol{b}\left(\times \mathbf{1 0}^{-\mathbf{1 0}}\right)$ & $\boldsymbol{c}\left(\times \mathbf{1 0}^{-\mathbf{1 2}}\right)$ & $\boldsymbol{a}\left(\times \mathbf{1 0}^{-\mathbf{7}}\right)$ & $\boldsymbol{b}\left(\times \mathbf{1 0}^{-\mathbf{1 0}}\right)$ & $\boldsymbol{c}\left(\times \mathbf{1 0}^{\mathbf{- 1 2}}\right)$ \\
\hline$\alpha=-2$ & 0.509 & -4.531 & 1.007 & 1.081 & -9.607 & 2.135 \\
$\alpha=1$ & 0.375 & -3.337 & 0.742 & 1.060 & -9.421 & 2.093 \\
$\alpha=2$ & 0.264 & -2.344 & 0.521 & 1.081 & -9.607 & 2.135 \\
$\alpha=5$ & 0.774 & -6.884 & 1.530 & 1.092 & -9.702 & 2.156 \\
\hline
\end{tabular}

In addition, we may plot the deflection curves of FGPM plates and FGM plates under various functional gradient indices, as shown in Figure 5, in which Figure 5a represents cases where $\alpha=-2,2$ and Figure $5 \mathrm{~b}$ represents cases where $\alpha=1,5$.

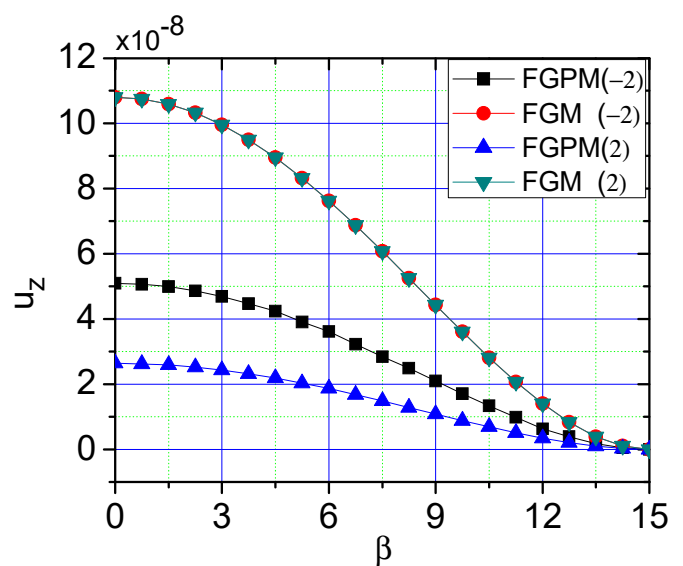

(a)

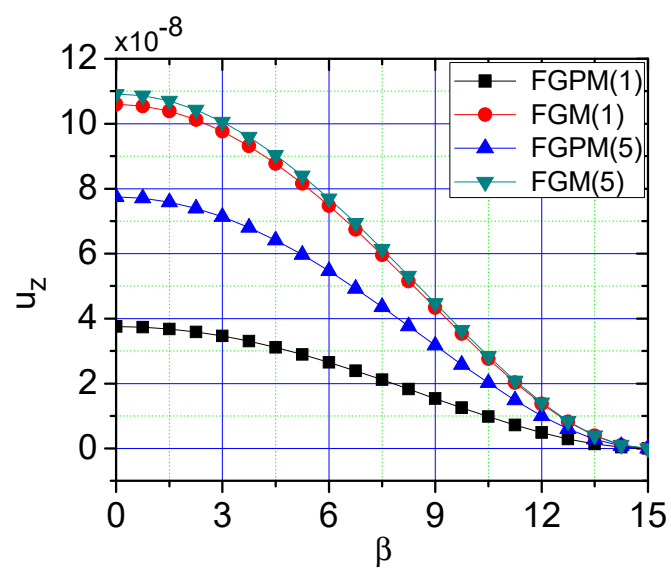

(b)

Figure 5. Deflection curves of FGPM plates and FGM plates: (a) $\alpha=-2$ and 2, (b) $\alpha=1$ and 5.

From Figure 5a,b, it is readily found that in cases where $\alpha=-2$ and 2, the curves FGM(-2) and FGM(2) basically coincide with each other; in cases where $\alpha=1$ and 5, the curves FGM(5) and FGM(1) are also pretty close. For the FGPM plates, this is not the case. This indicates that the change of functional gradient index may influence the deflection of FGPM plates to some extent, while for FGM plates, there seem to be no more differences when the gradient index varies.

It is worth noting that under the same functional gradient index, the deflection value of FGPM plates is smaller than that of corresponding FGM plates, which shows that the intervention of the piezoelectric effect may reduce the deformation of the plate. This phenomenon may be further explained from the point of view of energy conservation and transformation. For FGPM plates, a portion of the work done by applied external loads is transformed into electrical energy, due to the piezoelectric effect of materials; therefore, that portion transformed into elastic strain energy decreases correspondingly, and the deformation magnitude will be smaller than that of FGM plates, in which there is no so-called electrical energy transformation. 


\section{Conclusions}

In this study, we use a multi-parameter perturbation method to solve a functionally-graded, thin, circular piezoelectric plate under the action of uniformly-distributed loads, in which three piezoelectric coefficients, $D_{31}, D_{33}$, and $D_{15}$, are selected as perturbation parameters. The validity of the multi-parameter perturbation solution obtained was verified by numerical simulations based on layer-wise theory. The following three conclusions can be drawn:

(i) Adopting the three piezoelectric coefficients as perturbation parameters follows the basic idea of perturbation theory-i.e., if pure FGM plates without piezoelectric effects are taken as the undisturbed system, and the piezoelectric effect may be introduced as a kind of disturbance, then FGPM plates may be regarded as a disturbed system; thus, the solution for pure FGM plates may be easily obtained as a zero-order solution of the disturbed system.

(ii) In our perturbation, two stress functions and one electrical potential function were selected as basic functions. It was found that in the zero-order perturbation solution, only stress functions were not zero; thus, the zero-order solution actually corresponded to the elastic solution concerning elastic stress, elastic strain, and elastic displacement, this conclusion is consistent with the former conclusion: while in the first-order perturbation solution, only the electrical potential function was not zero; thus, the first-order solution actually corresponded to the electrical solution, concerning electrical potential, electrical field intensity, and electrical displacement.

(iii) The deformation magnitude of FGPM plates is generally smaller than that of corresponding FGM plates, showing the well-known piezoelectric stiffening effect. From the point of view of energy conservation and transformation, a portion of the work done by applied external loads is transformed into electrical energy, due to the piezoelectric effect, thus decreasing elastic strain energy stored in FGPM plates and resulting in the corresponding decrease in deformation magnitude.

It is undeniable that the calculation process of the multi-parameter perturbation method is somewhat cumbersome; this is the reason why we only calculate up to the first-order perturbation solution. If a higher accuracy of the solution is required, we need to resort to a higher order perturbation solution. However, the multi-parameter perturbation method proposed in this study may provide a reference for solving multi-physical field problems like electric, magnetic, or thermal fields, in addition to the traditional mechanical field. The theoretical result presented in this study is helpful to precisely analyze the mechanical properties of functionally-graded piezoelectric materials and structures, as well as to design sensors and actuators used extensively in electromechanical conversion. The related work is in progress.

Author Contributions: Conceptualization, X.-T.H. and J.-Y.S.; funding acquisition, X.-T.H. and J.-Y.S.; investigation, Y.-H.L.; methodology, Z.-X.Y.; software, Z.-X.Y. and Y.-H.L.; validation, X.L.; visualization, Y.-H.L. and X.L.; writing-original draft, X.-T.H. and Z.-X.Y.; writing-review and editing, X.L. and J.-Y.S. All authors have read and agreed to the published version of the manuscript.

Funding: This research was funded by the National Natural Science Foundation of China (Grant No. 11572061 and 11772072).

Conflicts of Interest: The authors declare no conflict of interest. The funders had no role in the design of the study; in the collection, analyses, or interpretation of data; in the writing of the manuscript, or in the decision to publish the results. 


\section{Appendix A Zero-Order Perturbation Solution}

Substituting the determined stress functions and the electrical potential function-i.e., $F_{1}^{(0)}, \psi_{1}^{(0)}$, and $\phi_{1}^{(0)}$ —into Equation (8), we have the zero-order stress solution

$$
\left\{\begin{array}{l}
\sigma_{r}=\frac{-12+4 S_{12}}{1+S_{12}} \beta^{2}\left(B_{1}^{(0)}+B_{2}^{(0)} \eta\right) e^{-\alpha \eta}+\frac{1}{\left(1+S_{12}\right)}\left\{\left[\frac{16 S_{13}\left(1-S_{12}\right)}{3\left(1+S_{12}\right)} B_{2}^{(0)} \eta^{3}+\frac{16 S_{13}\left(1-S_{12}\right)}{\left(1+S_{12}\right)} B_{1}^{(0)} \eta^{2}\right.\right. \\
\left.\left.-\frac{16 S_{44}}{\alpha\left(1+S_{12}\right)} B_{2}^{(0)} \eta^{2}+A_{5}^{(0)}+A_{6}^{(0)} \eta\right] e^{-\alpha \eta}-4 S_{13} A_{3}^{(0)} \eta-4 S_{13} A_{4}^{(0)}-\frac{2 S_{44}}{\alpha} A_{3}^{(0)}\right\} \\
\sigma_{\theta}=\frac{-4+12 S_{12}}{1+S_{12}} \beta^{2}\left(B_{1}^{(0)}+B_{2}^{(0)} \eta\right) e^{-\alpha \eta}+\frac{1}{\left(1+S_{12}\right)}\left\{\left[\frac{16 S_{13}\left(1-S_{12}\right)}{3\left(1+S_{12}\right)} B_{2}^{(0)} \eta^{3}+\frac{16 S_{13}\left(1-S_{12}\right)}{\left(1+S_{12}\right)} B_{1}^{(0)} \eta^{2}\right.\right. \\
\left.\left.-\frac{16 S_{44}}{\alpha\left(1+S_{12}\right)} B_{2}^{(0)} \eta^{2}+A_{5}^{(0)}+A_{6}^{(0)} \eta\right] e^{-\alpha \eta}-4 S_{13} A_{3}^{(0)} \eta-4 S_{13} A_{4}^{(0)}-\frac{2 S_{44}}{\alpha} A_{3}^{(0)}\right\} \\
\sigma_{z}=-\frac{64}{\left(1+S_{12}\right)}\left[\frac{1}{\alpha^{2}} B_{1}^{(0)}+\left(\frac{1}{\alpha^{2}} \eta+\frac{2}{\alpha^{3}}\right) B_{2}^{(0)}\right] e^{-\alpha \eta}+4 A_{3}^{(0)} \eta+4 A_{4}^{(0)} \\
\tau_{r z}=\frac{q \beta}{-e^{\alpha}+e^{-\alpha}+2 \alpha}\left\{e^{-\alpha / 2-\alpha \eta\left[\frac{\left(4 \alpha-\alpha^{2}\right)}{8}-\alpha \eta+\frac{\alpha^{2}}{2} \eta^{2}\right]}\right. \\
\left.\left.+e^{\alpha / 2-\alpha \eta\left[\frac{4 \alpha+\alpha^{2}}{8}\right.}+\alpha \eta-\frac{\alpha^{2}}{2} \eta^{2}\right]-\alpha\right\}
\end{array}\right.
$$

the zero-order strain solutions

$$
\left\{\begin{array}{l}
\varepsilon_{r}=\beta^{2}\left(-12+12 S_{12}\right)\left(B_{1}^{(0)}+B_{2}^{(0)} \eta\right)+\frac{16 S_{13}\left(1-S_{12}\right)}{3\left(1+S_{12}\right)} B_{2}^{(0)} \eta^{3}+\frac{16 S_{13}\left(1-S_{12}\right)}{\left(1+S_{12}\right)} B_{1}^{(0)} \eta^{2} \\
-\frac{16 S_{44}}{\alpha\left(1+S_{12}\right)} B_{2}^{(0)} \eta^{2}+A_{5}^{(0)}+A_{6}^{(0)} \eta-\frac{64 S_{13}}{\left(1+S_{12}\right.}\left[\frac{1}{\alpha^{2}} B_{1}^{(0)}+\left(\frac{1}{\alpha^{2}} \eta+\frac{2}{\alpha^{3}}\right) B_{2}^{(0)}\right]-\frac{2 S_{44}}{\alpha} A_{3}^{(0)} e^{\alpha \eta} \\
\varepsilon_{\theta}=\beta^{2}\left(-4+4 S_{12}\right)\left(B_{1}^{(0)}+B_{2}^{(0)} \eta\right)+\frac{16 S_{13}\left(1-S_{12}\right)}{3\left(1+S_{12}\right)} B_{2}^{(0)} \eta^{3}+\frac{16 S_{13}\left(1-S_{12}\right)}{\left(1+S_{12}\right)} B_{1}^{(0)} \eta^{2} \\
-\frac{16 S_{44}}{\alpha\left(1+S_{12}\right)} B_{2}^{(0)} \eta^{2}+A_{5}^{(0)}+A_{6}^{(0)} \eta-\frac{64 S_{13}}{\left(1+S_{12}\right)}\left[\frac{1}{\alpha^{2}} B_{1}^{(0)}+\left(\frac{1}{\alpha^{2}} \eta+\frac{2}{\alpha^{3}}\right) B_{2}^{(0)}\right]-\frac{2 S_{44}}{\alpha} A_{3}^{(0)} e^{\alpha \eta} \\
\varepsilon_{z}=\frac{16 S_{13}\left(S_{12}-1\right)}{\left(1+S_{12}\right)} \beta^{2}\left(B_{1}^{(0)}+B_{2}^{(0)} \eta\right)+\frac{2 S_{13}}{\left(1+S_{12}\right)}\left[\frac{16 S_{13}\left(1-S_{12}\right)}{3\left(1+S_{12}\right)} B_{2}^{(0)} \eta^{3}+\frac{16 S_{13}\left(1-S_{12}\right)}{\left(1+S_{12}\right)} B_{1}^{(0)} \eta^{2}\right. \\
\left.-\frac{16 S_{44}}{\alpha\left(1+S_{12}\right)} B_{2}^{(0)} \eta^{2}+A_{5}^{(0)}+A_{6}^{(0)} \eta\right]+\left[\frac{2 S_{13}}{\left(1+S_{12}\right)}\left(-4 S_{13} A_{3}^{(0)} \eta-4 S_{13} A_{4}^{(0)}-\frac{2 S_{44}}{\alpha} A_{3}^{(0)}\right)\right. \\
\left.+4 S_{33} A_{3}^{(0)} \eta+4 S_{33} A_{4}^{(0)}\right] e^{\alpha \eta}-\frac{64 S_{33}}{\left(1+S_{12}\right)}\left[\frac{1}{\alpha^{2}} B_{1}^{(0)}+B_{2}^{(0)}\left(\frac{1}{\alpha^{2}} \eta+\frac{2}{\alpha^{3}}\right)\right]
\end{array}\right.
$$

the zero-order electrical displacements and electrical strength solutions

$$
\left\{\begin{array}{l}
D_{r}=e^{\alpha \eta} D_{15} \tau_{r z} \\
D_{z}=e^{\alpha \eta}\left(D_{31} \sigma_{r}+D_{31} \sigma_{\theta}+D_{33} \sigma_{z}\right) \\
E_{r}=0 \\
E_{z}=0
\end{array}\right.
$$

as well as the radial displacement solution

$$
\begin{aligned}
& u_{r}=\beta^{3}\left(-4+4 S_{12}\right)\left(B_{1}^{(0)}+B_{2}^{(0)} \eta\right)+\beta\left\{\frac{16 S_{13}\left(1-S_{12}\right)}{3\left(1+S_{12}\right)} B_{2}^{(0)} \eta^{3}\right. \\
& +\frac{16 S_{13}\left(1-S_{12}\right)}{\left(1+S_{12}\right)} B_{1}^{(0)} \eta^{2}-\frac{16 S_{44}}{\alpha\left(1+S_{12}\right)} B_{2}^{(0)} \eta^{2}+A_{5}^{(0)}+A_{6}^{(0)} \eta \\
& \left.-\frac{64 S_{13}}{\left(1+S_{12}\right)}\left[\frac{1}{\alpha^{2}} B_{1}^{(0)}+\left(\frac{1}{\alpha^{2}} \eta+\frac{2}{\alpha^{3}}\right) B_{2}^{(0)}\right]-\frac{2 S_{44}}{\alpha} A_{3}^{(0)} e^{\alpha \eta}\right\}
\end{aligned}
$$

Note that $A_{5}^{(0)}$ and $A_{6}^{(0)}$, as well as $z$-direction displacement $u_{z}$, are still not determined. Next, we will derive the numerical value solution of $A_{5}^{(0)}$ and $A_{6}^{(0)}$ in different gradient indexes.

(1) Determination of the unknown neutral layer $\eta_{n}$

In this study, it is assumed that the so-called neutral layer means the layer at $u_{r}=0$. For a thin plate under small-deflection bending, the neutral layer exists in the plate, and does not move out of the 
plate. To solve the concrete location, we use $u_{r}=r \cdot \varepsilon_{\theta}$ in Equation (3). For any $\beta$, we have $\varepsilon_{\theta}(\beta, \eta)=0$; thus, the second equation of Equation (A2) will give

$$
\left\{\begin{array}{l}
B_{1}^{(0)}+B_{2}^{(0)} \eta_{n}=0 \\
\frac{16 S_{13}\left(1-S_{12}\right)}{3\left(1+S_{12}\right)} B_{2}^{(0)} \eta_{n}^{3}+\frac{16 S_{13}\left(1-S_{12}\right)}{\left(1+S_{12}\right)} B_{1}^{(0)} \eta_{n}^{2}-\frac{16 S_{44}}{\alpha\left(1+S_{12}\right)} B_{2}^{(0)} \eta_{n}^{2}+A_{5}^{(0)} \\
+A_{6}^{(0)} \eta_{n}-\frac{2 S_{44}}{\alpha} A_{3}^{(0)} e^{\alpha \eta_{n}}-\frac{64 S_{13}}{\left(1+S_{12}\right)}\left[\frac{1}{\alpha^{2}} B_{1}^{(0)}+\left(\frac{1}{\alpha^{2}} \eta_{n}+\frac{2}{\alpha^{3}}\right) B_{2}^{(0)}\right]=0
\end{array}\right.
$$

Lastly, we obtain

$$
\eta_{n}=-\frac{B_{1}^{(0)}}{B_{2}^{(0)}}
$$

which may be used for the solution of unknown neutral layer $\eta_{n}$. Table A1 gives values of $\eta_{n}$ in different gradient indexes.

Table A1. Value of $\eta_{n}$ in different gradient indexes.

\begin{tabular}{ccccc}
\hline Gradient Index & $\alpha=-2$ & $\alpha=0$ & $\alpha=2$ & $\alpha=5$ \\
\hline$\eta_{n}$ & 0.157 & 0 & -0.157 & -0.307 \\
\hline
\end{tabular}

(2) Determination of the $z$-direction displacement $u_{z}$

From the latter three expressions of Equation (3), we have

$$
\left\{\begin{array}{l}
u_{z}=\int \varepsilon_{z}(\beta, \eta) d \eta=m(\beta, \eta)+n(\beta) \\
u_{r}=\beta \varepsilon_{\theta}(\beta, \eta) \\
\gamma_{r z}=\frac{\partial u_{z}}{\partial \beta}+\frac{\partial u_{r}}{\partial \eta}=\frac{\partial[m(\beta, \eta)+n(\beta)]}{\partial \beta}+\frac{\partial\left[\beta \cdot \varepsilon_{\theta}(\beta, \eta)\right]}{\partial \eta}
\end{array}\right.
$$

where $\varepsilon_{z}(\beta, \eta)$ and $\varepsilon_{\theta}(\beta, \eta)$ are zero-order strains, and $m(\beta, \eta)$ and $n(\beta)$ are two unknown functions. By using the fourth expression of Equation (4), and also noting that $E_{r}=0$, we have

$$
\gamma_{r z}=e^{\alpha z / h} S_{44} \tau_{r z}(\beta, \eta)
$$

where $\tau_{r z}(\beta, \eta)$ is the zero-order stress. By letting $\gamma_{r z}$ in Equation (A7) be equal to $\gamma_{r z}$ in Equation (A8), we have

$$
\frac{\partial[m(\beta, \eta)+n(\beta)]}{\partial \beta}+\frac{\partial\left[\beta \varepsilon_{\theta}(\beta, \eta)\right]}{\partial \eta}=e^{\alpha z / h} S_{44} \tau_{r z}(\beta, \eta)
$$

Thus, we may obtain the following relationship:

$$
\beta l_{1}(\eta)+\beta^{3} l_{2}(\eta)=\frac{d n(\beta)}{d \beta}
$$

Where $l_{1}(\eta)$ and $l_{2}(\eta)$ are two functions of $\eta$. By letting $\eta=\eta_{n}$ in Equation (A10), we may obtain $n(\beta)$, containing $A_{5}^{(0)}$ and $A_{6}^{(0)}$. Substituting this $n(\beta)$ into $u_{z}$ in Equation (A7), and also considering the second expression in Equation (A5) and boundary conditions of the fixed periphery in Equation (33), we may obtain the $n(\beta)$ in which $A_{5}^{(0)}$ and $A_{6}^{(0)}$ are determined. Thus, $A_{5}^{(0)}$ and $A_{6}^{(0)}$ as well as z-direction displacement $u_{z}$, are finally determined. By adopting this method, we may compute the values of $A_{5}^{(0)}$ and $A_{6}^{(0)}$ in different gradient indexes, as shown in Table A2. 
Table A2. $A_{5}^{(0)}$ and $A_{6}^{(0)}$ in different gradient indexes.

\begin{tabular}{ccccc}
\hline Gradient Index & $\alpha=-2$ & $\alpha=1$ & $\alpha=2$ & $\alpha=5$ \\
\hline$A_{5}^{(0)}\left(\times 10^{10}\right)$ & -2.517 & -2.070 & 2.517 & -1.252 \\
$A_{6}^{(0)}\left(\times 10^{10}\right)$ & 1.830 & 1.647 & 1.830 & 1.879 \\
\hline
\end{tabular}

\section{Appendix B First-Order Perturbation Solution}

Substituting Equation (20) into Equations (35)-(37) for $\mathrm{D}_{31}$, we may have three equations of $f_{11}^{(1)}(\eta), f_{12}^{(1)}(\eta), f_{13}^{(1)}(\eta), g_{11}^{(1)}(\eta), g_{12}^{(1)}(\eta), h_{11}^{(1)}(\eta), h_{12}^{(1)}(\eta)$, and $h_{13}^{(1)}(\eta)$. The satisfaction for these equations will give the conditions that the same power terms of $\beta$ are uniformly zero; thus, we obtain

$$
\begin{aligned}
& \left\{\begin{array}{l}
\beta^{5}: 2 \alpha \frac{d^{2} f_{11}^{(0)}}{d \eta^{2}}+2 \frac{d^{3} f_{11}^{(0)}}{d \eta^{3}}-\alpha \lambda_{33} \frac{d h_{11}^{(1)}}{d \eta}-\lambda_{33} \frac{d^{2} h_{11}^{(1)}}{d \eta^{2}}=0 \\
\beta^{3}: 2 \alpha \frac{d^{2} f_{12}^{(0)}}{d \eta^{2}}+2 \frac{d^{3} f_{12}^{(0)}}{d \eta^{3}}+16 \alpha g_{11}^{(0)}+16 \frac{d g_{11}^{(0)}}{d \eta}-16 h_{11}^{(1)}-\alpha \lambda_{33} \frac{d h_{12}^{(1)}}{d \eta}-\lambda_{33} \frac{d^{2} h_{12}^{(1)}}{d \eta^{2}}=0 \\
\beta: 2 \alpha \frac{d^{2} f_{13}^{(0)}}{d \eta^{2}}+2 \frac{d^{3} f_{13}^{(0)}}{d \eta^{3}}+4 \alpha g_{12}^{(0)}+4 \frac{d g_{12}^{(0)}}{d \eta}-4 h_{12}^{(1)}-\alpha \lambda_{33} \frac{d h_{13}^{(1)}}{d \eta}-\lambda_{33} \frac{d^{2} h_{13}^{(1)}}{d \eta^{2}}=0 \\
+\alpha^{2}\left(1+S_{12}\right) \frac{d^{2} f_{13}^{(1)}}{d \eta^{2}}+2 \alpha\left(1+S_{12}\right) \frac{d^{3} f_{13}^{(1)}}{d \eta^{3}}+\left(1+S_{12}\right) \frac{d^{4} f_{13}^{(1)}}{d \eta^{4}}+32 S_{13} g_{11}^{(1)} \\
+2 \alpha^{2}\left(1+S_{12}\right) g_{12}^{(1)}+4 \alpha\left(1+S_{12}\right) \frac{d g_{12}^{(1)}}{d \eta}+2\left(1+S_{12}\right) \frac{d^{2} g_{12}^{(1)}}{d \eta^{2}}-\alpha^{2} \frac{d h_{13}^{(0)}}{d \eta} \\
-2 \alpha \frac{d^{2} h_{13}^{(0)}}{d \eta^{2}}-\frac{d^{3} h_{13}^{(0)}}{d \eta^{3}}=0 \\
\beta^{3}: 16 \alpha^{2} S_{13} f_{11}^{(1)}+4 \alpha\left(8 S_{13}+3 S_{44}\right) \frac{d f_{11}^{(1)}}{d \eta}+4\left(10 S_{13}+3 S_{44}\right) \frac{d^{2} f_{11}^{(1)}}{d \eta^{2}}+\alpha^{2}\left(1+S_{12}\right) \\
\frac{d^{2} f_{12}^{(1)}}{d \eta^{2}}+2 \alpha\left(1+S_{12}\right) \frac{d^{3} f_{12}^{(1)}}{d \eta^{3}}+\left(1+S_{12}\right) \frac{d^{4} f_{12}^{(1)}}{d \eta^{4}}+4 \alpha^{2}\left(1+3 S_{12}\right) g_{11}^{(1)}+8 \alpha \\
\left(1+3 S_{12}\right) \frac{d g_{11}^{(1)}}{d \eta}+4\left(1+3 S_{12}\right) \frac{d^{2} g_{11}^{(1)}}{d \eta^{2}}-\alpha^{2} \frac{d h_{12}^{(0)}}{d \eta}-2 \alpha \frac{d^{2} h_{12}^{(0)}}{d \eta^{2}}-\frac{d^{3} h_{12}^{(0)}}{d \eta^{3}}=0 \\
\beta^{7}: \alpha^{2}\left(1+S_{12}\right) \frac{d^{2} f_{11}^{(1)}}{d \eta^{2}}+2 \alpha\left(1+S_{12}\right) \frac{d^{3} f_{11}^{(1)}}{d \eta^{3}}+\left(1+S_{12}\right) \frac{d^{4} f_{11}^{(1)}}{d \eta^{4}}-\alpha^{2} \frac{d h_{11}^{(0)}}{d \eta} \\
-2 \alpha \frac{d^{2} h_{11}^{(0)}}{d \eta^{2}}-\frac{d^{3} h_{11}^{(0)}}{d \eta^{3}}=0
\end{array}\right.
\end{aligned}
$$


Solving the above three equations, we have

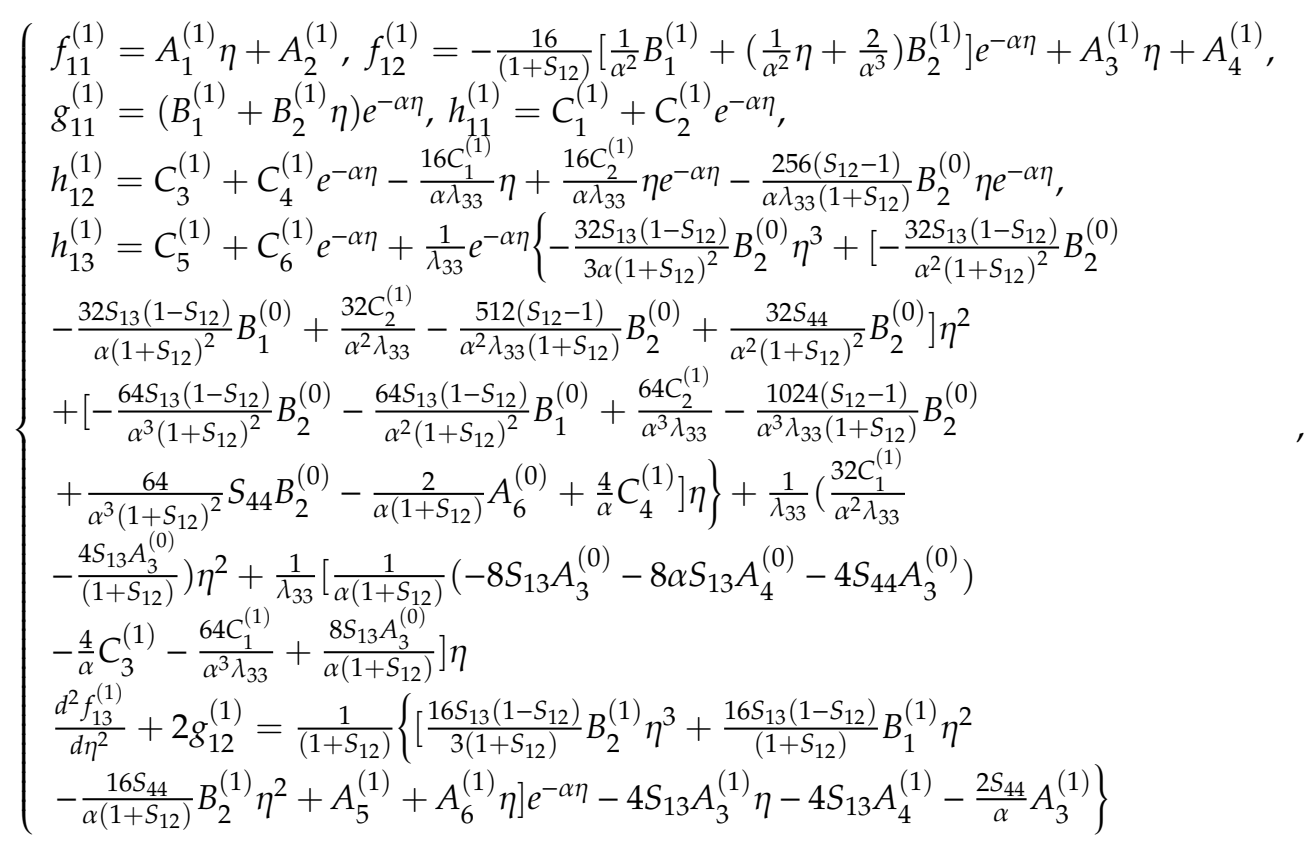

where $A_{1}^{(1)}, A_{2}^{(1)}, A_{3}^{(1)}, A_{4}^{(1)}, A_{5}^{(1)}, A_{6}^{(1)}, B_{1}^{(1)}, B_{2}^{(1)}, C_{1}^{(1)}, C_{2}^{(1)}, C_{3}^{(1)}, C_{4}^{(1)}, C_{5}^{(1)}$, and $C_{6}^{(1)}$ are integral constants of the first-order perturbation to $D_{31}$. By using boundary conditions (38) and (39), these constants are determined as

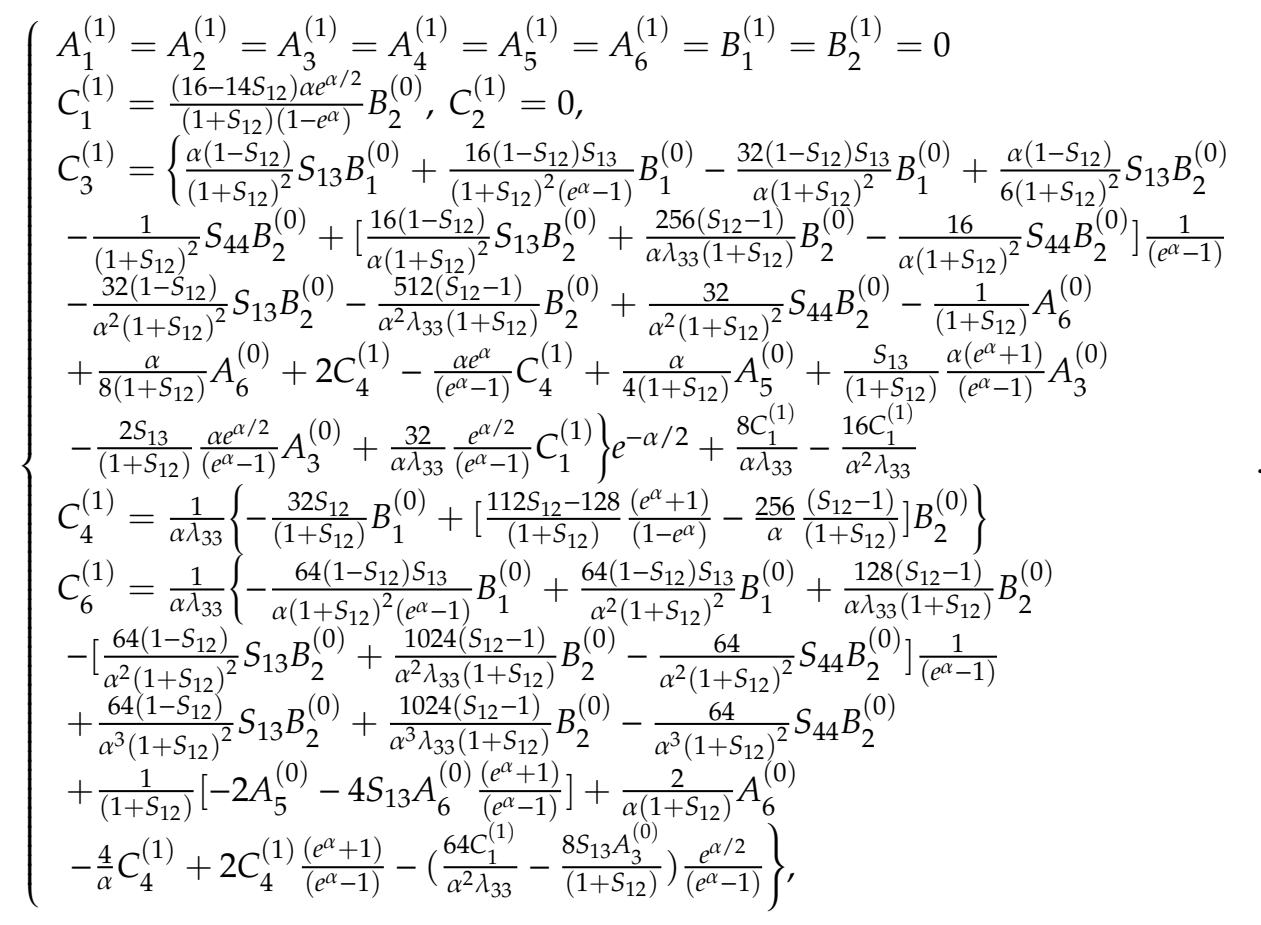

Note that there is no need to determine $C_{5}^{(1)}$, since it is the constant term of electrical potential function and has no effect on the electrical field.

Similarly, for $D_{33}$, substituting Equation (20) into Equations (40)-(42), we may have three equations of $f_{21}^{(1)}(\eta), f_{22}^{(1)}(\eta), f_{23}^{(1)}(\eta), g_{21}^{(1)}(\eta), g_{22}^{(1)}(\eta), h_{21}^{(1)}(\eta), h_{22}^{(1)}(\eta)$, and $h_{23}^{(1)}(\eta)$. The satisfaction for 
these equations will give the conditions that the same power terms of $\beta$ are uniformly zero; thus, we obtain

$$
\begin{aligned}
& \left\{\begin{array}{l}
\beta^{5}: 4 \alpha f_{12}^{(0)}+4 \frac{d f_{12}^{(0)}}{d \eta}-4 h_{22}^{(1)}-\lambda_{33} \frac{d^{2} h_{23}^{(1)}}{d \eta^{2}}-\alpha \lambda_{33} \frac{d h_{23}^{(1)}}{d \eta}=0 \\
\beta^{3}: 4 \alpha f_{11}^{(0)}+12 \alpha f_{11}^{(0)}+16 \frac{d f_{11}^{(0)}}{d \eta}-16 h_{21}^{(1)}-\lambda_{33} \frac{d^{2} h_{22}^{(1)}}{d \eta^{2}}-\alpha \lambda_{33} \frac{d h_{22}^{(1)}}{d \eta}=0 \\
\beta:-\lambda_{33} \frac{d^{2} h_{21}^{(1)}}{d \eta^{2}}-\alpha \lambda_{33} \frac{d h_{21}^{(1)}}{d \eta}=0
\end{array},\right. \\
& \left\{\begin{array}{l}
\beta^{3}: 32 S_{33} f_{21}^{(1)}+4 \alpha^{2} S_{13} f_{22}^{(1)}+2 \alpha\left(4 S_{13}+S_{44}\right) \frac{d f_{22}^{(1)}}{d \eta}+2\left(4 S_{13}+S_{44}\right) \frac{d^{2} f_{22}^{(1)}}{d \eta^{2}} \\
+\alpha^{2}\left(1+S_{12}\right) \frac{d^{2} f_{23}^{(1)}}{d \eta^{2}}+2 \alpha\left(1+S_{12}\right) \frac{d^{3} f_{23}^{(1)}}{d \eta^{3}}+\left(1+S_{12}\right) \frac{d^{4} f_{23}^{(1)}}{d \eta^{4}}+32 S_{13} g_{21}^{(1)} \\
+2 \alpha^{2}\left(1+S_{12}\right) g_{22}^{(1)}+4 \alpha\left(1+S_{12}\right) \frac{d g_{22}^{(1)}}{d \eta}+2\left(1+S_{12}\right) \frac{d^{2} g_{22}^{(1)}}{d \eta^{2}}-2 \frac{d h_{12}^{(0)}}{d \eta}=0 \\
\beta^{5}: 16 \alpha^{2} S_{13} f_{21}^{(1)}+4 \alpha\left(8 S_{13}+3 S_{44}\right) \frac{d f_{21}^{(1)}}{d \eta}+4\left(10 S_{13}+3 S_{44}\right) \frac{d^{2} f_{21}^{(1)}}{d \eta^{2}} \\
+\alpha^{2}\left(1+S_{12}\right) \frac{d^{2} f_{22}^{(1)}}{d \eta^{2}}+2 \alpha\left(1+S_{12}\right) \frac{d^{3} f_{22}^{(1)}}{d \eta^{3}}+\left(1+S_{12}\right) \frac{d^{4} f_{22}^{(1)}}{d \eta^{4}} \\
+4 \alpha^{2}\left(1+3 S_{12}\right) g_{21}^{(1)}+8 \alpha\left(1+3 S_{12}\right) \frac{d g_{21}^{(1)}}{d \eta}+4\left(1+3 S_{12}\right) \frac{d^{2} g_{21}^{(1)}}{d \eta^{2}}-12 \frac{d h_{11}^{(0)}}{d \eta}=0 \\
\beta^{7}: \alpha^{2}\left(1+S_{12}\right) \frac{d^{2} f_{21}^{(1)}}{d \eta^{2}}+2 \alpha\left(1+S_{12}\right) \frac{d^{3} f_{21}^{(1)}}{d \eta^{3}}+\left(1+S_{12}\right) \frac{d^{4} f_{21}^{(1)}}{d \eta^{4}}=0
\end{array}\right. \\
& \left\{\begin{array}{l}
\beta^{3}: 32 S_{13} f_{21}^{(1)}+2\left(1+S_{12}\right) \frac{d^{2} f_{22}^{(1)}}{d \eta^{2}}+32 g_{21}^{(1)}=0 \\
\beta^{5}: 4\left(1+S_{12}\right) \frac{d^{2} f_{21}^{(1)}}{d \eta^{2}}=0
\end{array} .\right.
\end{aligned}
$$

Solving the above three equations, we have

$$
\left\{\begin{array}{l}
f_{21}^{(1)}=A_{7}^{(1)} \eta+A_{8}^{(1)}, f_{22}^{(1)}=-\frac{16}{\left(1+S_{12}\right)}\left[\frac{1}{\alpha^{2}} B_{3}^{(1)}+B_{4}^{(1)}\left(\frac{1}{\alpha^{2}} \eta+\frac{2}{\alpha^{3}}\right)\right] e^{-\alpha \eta}+A_{9}^{(1)} \eta+A_{10}^{(1)}, \\
g_{21}^{(1)}=\left(B_{3}^{(1)}+B_{4}^{(1)} \eta\right) e^{-\alpha \eta}, h_{21}^{(1)}=C_{7}^{(1)}+C_{8}^{(1)} e^{-\alpha \eta}, h_{22}^{(1)}=C_{9}^{(1)}+C_{10}^{(1)} e^{-\alpha \eta}-\frac{16 C_{7}^{(1)}}{\alpha \lambda_{33}} \eta+\frac{16 C_{8}^{(1)}}{\alpha \lambda_{33}} \eta e^{-\alpha \eta}, \\
h_{23}^{(1)}=C_{11}^{(1)}+C_{12}^{(1)} e^{-\alpha \eta}+\frac{e^{-\alpha \eta}}{\lambda_{33}}\left[\frac{32 C_{8}^{(1)}}{\alpha^{2} \lambda_{33}} \eta^{2}+\left(\frac{64 C_{8}^{(1)}}{\alpha^{3} \lambda_{33}}+\frac{64}{\alpha^{3}\left(1+S_{12}\right)} B_{2}^{(0)}+\frac{4}{\alpha} C_{10}^{(1)}\right) \eta\right] \\
+\frac{1}{\lambda_{33}}\left(2 A_{3}^{(0)}+\frac{32 C_{7}^{(1)}}{\alpha^{2} \lambda_{33}}\right) \eta^{2}+\frac{1}{\lambda_{33}}\left(4 A_{4}^{(0)}-\frac{4}{\alpha} C_{9}^{(1)}-\frac{64 C_{7}^{(1)}}{\alpha^{3} \lambda_{33}}\right) \eta \\
\frac{d^{2} f_{23}^{(1)}}{d \eta^{2}}+2 g_{22}^{(1)}=\frac{1}{\left(1+S_{12}\right)}\left\{\left[\frac{16 S_{13}\left(1-S_{12}\right)}{3\left(1+S_{12}\right)} B_{4}^{(1)} \eta^{3}+\frac{16 S_{13}\left(1-S_{12}\right)}{\left(1+S_{12}\right)} B_{3}^{(1)} \eta^{2}\right.\right. \\
\left.\left.\quad-\frac{16 S_{44}}{\alpha\left(1+S_{12}\right)} B_{4}^{(1)} \eta^{2}+A_{11}^{(1)}+A_{12}^{(1)} \eta\right] e^{-\alpha \eta}-4 S_{13} A_{9}^{(1)} \eta-4 S_{13} A_{10}^{(1)}-\frac{2 S_{44}}{\alpha} A_{9}^{(1)}\right\}
\end{array}\right.
$$

where $A_{7}^{(1)}, A_{8}^{(1)}, A_{9}^{(1)}, A_{10}^{(1)}, A_{11}^{(1)}, A_{12}^{(1)}, B_{3}^{(1)}, B_{4}^{(1)}, C_{7}^{(1)}, C_{8}^{(1)}, C_{9}^{(1)}, C_{10}^{(1)}, C_{11}^{(1)}$, and $C_{12}^{(1)}$ are integral constants of the first-order perturbation to $D_{33}$. By using boundary conditions (43) and (44), these constants are determined as

$$
\left\{\begin{array}{l}
A_{7}^{(1)}=A_{8}^{(1)}=A_{9}^{(1)}=A_{10}^{(1)}=A_{11}^{(1)}=A_{12}^{(1)}=0, \\
B_{3}^{(1)}=B_{4}^{(1)}=0 \\
C_{7}^{(1)}=C_{8}^{(1)}=C_{9}^{(1)}=C_{10}^{(1)}=0 \\
C_{12}^{(1)}=\frac{64}{\alpha^{3} \lambda_{33}\left(1+S_{12}\right)} B_{1}^{(0)}+\frac{384}{\alpha^{4} \lambda_{33}\left(1+S_{12}\right)} B_{2}^{(0)}
\end{array} .\right.
$$

Note that there is also no need to determine $C_{11}^{(1)}$.

Similarly, for D15, substituting Equation (20) into Equation (45)-(47), we may have three equations of $f_{31}^{(1)}(\eta), f_{32}^{(1)}(\eta), f_{33}^{(1)}(\eta), g_{31}^{(1)}(\eta), g_{32}^{(1)}(\eta), h_{31}^{(1)}(\eta), h_{32}^{(1)}(\eta)$, and $h_{33}^{(1)}(\eta)$. The satisfaction for 
these equations will give the conditions that the same power terms of $\beta$ are uniformly zero; thus, we obtain

$$
\begin{gathered}
\left\{\begin{array}{l}
\beta^{5}: 4 \frac{d f_{12}^{(0)}}{d \eta}+4 h_{32}^{(1)}+\lambda_{33} \frac{d^{2} h_{33}^{(1)}}{d \eta^{2}}+\alpha \lambda_{33} \frac{d h_{33}^{(1)}}{d \eta}=0 \\
\beta^{3}: 16 \frac{d f_{11}^{(0)}}{d \eta}+4 \frac{d f_{12}^{(0)}}{d \eta}+16 h_{31}^{(1)}+\lambda_{33} \frac{d^{2} h_{32}^{(1)}}{d \eta^{2}}+\alpha \lambda_{33} \frac{d h_{32}^{(1)}}{d \eta}=0, \\
\beta: \lambda_{33} \frac{d^{2} h_{31}^{(1)}}{d \eta^{2}}+\alpha \lambda_{33} \frac{d h_{31}^{(1)}}{d \eta}=0
\end{array}\right. \\
\left\{\begin{array}{l}
\beta^{3}: 32 S_{33} f_{31}^{(1)}+4 \alpha^{2} S_{13} f_{32}^{(1)}+2 \alpha\left(4 S_{13}+S_{44}\right) \frac{d f_{32}^{(1)}}{d \eta}+2\left(4 S_{13}+S_{44}\right) \frac{d^{2} f_{32}^{(1)}}{d \eta^{2}} \\
+\alpha^{2}\left(1+S_{12}\right) \frac{d^{2} f_{33}^{(1)}}{d \eta^{2}}+2 \alpha\left(1+S_{12}\right) \frac{d^{3} f_{33}^{(1)}}{d \eta^{3}}+\left(1+S_{12}\right) \frac{d^{4} f_{33}^{(1)}}{d \eta^{4}}+32 S_{13} g_{31}^{(1)} \\
+2 \alpha^{2}\left(1+S_{12}\right) g_{32}^{(1)}+4 \alpha\left(1+S_{12}\right) \frac{d g_{32}^{(1)}}{d \eta}+2\left(1+S_{12}\right) \frac{d^{2} g_{32}^{(1)}}{d \eta^{2}}+2 \alpha h_{12}^{(0)}+2 \frac{d h_{12}^{(0)}}{d \eta}=0 \\
\beta^{5}: 16 \alpha^{2} S_{13} f_{31}^{(1)}+4 \alpha\left(8 S_{13}+3 S_{44}\right) \frac{d f_{31}^{(1)}}{d \eta}+4\left(10 S_{13}+3 S_{44}\right) \frac{d^{2} f_{31}^{(1)}}{d \eta^{2}} \\
+\alpha^{2}\left(1+S_{12}\right) \frac{d^{2} f_{32}^{(1)}}{d \eta^{2}}+2 \alpha\left(1+S_{12}\right) \frac{d^{3} f_{32}^{(1)}}{d \eta^{3}}+\left(1+S_{12}\right) \frac{d^{4} f_{32}^{(1)}}{d \eta^{4}}+4 \alpha^{2}\left(1+3 S_{12}\right) g_{31}^{(1)} \\
+8 \alpha\left(1+3 S_{12}\right) \frac{d g_{31}^{(1)}}{d \eta}+4\left(1+3 S_{12}\right) \frac{d^{2} g_{31}^{(1)}}{d \eta^{2}}+12 \alpha h_{11}^{(0)}+12 \frac{d h_{11}^{(0)}}{d \eta}=0 \\
\beta^{7}: \alpha^{2}\left(1+S_{12}\right) \frac{d^{2} f_{31}^{(1)}}{d \eta^{2}}+2 \alpha\left(1+S_{12}\right) \frac{d^{3} f_{31}^{(1)}}{d \eta^{3}}+\left(1+S_{12}\right) \frac{d^{4} f_{31}^{(1)}}{d \eta^{4}}=0 \\
\beta^{3}: 32 S_{13} f_{31}^{(1)}+2\left(1+S_{12}\right) \frac{d^{2} f_{32}^{(1)}}{d \eta^{2}}+32 g_{31}^{(1)}=0 \\
\beta^{5}: 4\left(1+S_{12}\right) \frac{d^{2} f_{31}^{(1)}}{d \eta^{2}}=0
\end{array},\right.
\end{gathered}
$$

Solving the above three equations, we have

$$
\left\{\begin{array}{l}
f_{31}^{(1)}=A_{13}^{(1)} \eta+A_{14}^{(1)}, f_{32}^{(1)}=-\frac{16}{\left(1+S_{12}\right)}\left[\frac{1}{\alpha^{2}} B_{3}^{(1)}+B_{4}^{(1)}\left(\frac{1}{\alpha^{2}} \eta+\frac{2}{\alpha^{3}}\right)\right] e^{-\alpha \eta}+A_{15}^{(1)} \eta+A_{16}^{(1)}, \\
g_{31}^{(1)}=\left(B_{5}^{(1)}+B_{6}^{(1)} \eta\right) e^{-\alpha \eta}, h_{31}^{(1)}=C_{13}^{(1)}+C_{14}^{(1)} e^{-\alpha \eta}, \\
h_{32}^{(1)}=C_{15}^{(1)}+C_{16}^{(1)} e^{-\alpha \eta}+\frac{1}{\lambda_{33}}\left[\frac{32}{\alpha^{2}\left(1+S_{12}\right)} B_{2}^{(0)} \eta^{2}+\left(\frac{128}{\alpha^{3}\left(1+S_{12}\right)} B_{2}^{(0)}+\frac{64}{\alpha^{2}\left(1+S_{12}\right)} B_{1}^{(0)}\right.\right. \\
\left.\left.+\frac{16}{\alpha} C_{14}^{(1)}\right) \eta\right] e^{-\alpha \eta}+\frac{-4 A_{3}^{(0)}-16 C_{13}^{(1)}}{\alpha \lambda_{33}} \eta \\
h_{33}^{(1)}=C_{17}^{(1)}+C_{18}^{(1)} e^{-\alpha \eta}+\frac{e^{-\alpha \eta}}{\lambda_{33}}\left[\frac{128}{3 \alpha^{3} \lambda_{33}\left(1+S_{12}\right)} B_{2}^{(0)} \eta^{3}+\left(\frac{384}{\alpha^{4} \lambda_{33}\left(1+S_{12}\right)} B_{2}^{(0)}+\frac{64}{\alpha^{3} \lambda_{33}\left(1+S_{12}\right)} B_{1}^{(0)}\right.\right. \\
\left.+\frac{32}{\alpha^{2} \lambda_{33}} C_{14}^{(1)}+\frac{32}{\alpha^{2}\left(1+S_{12}\right)} B_{2}^{(0)}\right) \eta^{2}+\left(\frac{768}{\alpha^{5} \lambda_{33}\left(1+S_{12}\right)} B_{2}^{(0)}+\frac{128}{\alpha^{4} \lambda_{33}\left(1+S_{12}\right)} B_{1}^{(0)}+\frac{64}{\alpha^{3} \lambda_{33}} C_{14}^{(1)}\right. \\
\left.\left.+\frac{128}{\alpha^{3}\left(1+S_{12}\right)} B_{2}^{(0)}+\frac{64}{\alpha^{2}\left(1+S_{12}\right)} B_{1}^{(0)}+\frac{4}{\alpha} C_{16}^{(1)}\right) \eta\right]+\frac{8 A_{3}+32 C_{13}^{(1)}}{\alpha^{2} \lambda_{33}^{2}} \eta^{2}+\left(\frac{-4 A_{3}^{(0)}-4 C_{15}^{(1)}}{\alpha \lambda_{33}}\right. \\
-\frac{16 A_{3}^{(0)}+64 C_{13}^{(1)}}{\alpha^{3} \lambda_{33}^{2}} \eta \eta \\
\frac{d^{2} f_{33}^{(1)}}{d \eta^{2}}+2 g_{32}^{(1)}=\frac{1}{\left(1+S_{12}\right)}\left\{\left[\frac{16 S_{13}\left(1-S_{12}\right)}{3\left(1+S_{12}\right)} B_{6}^{(1)} \eta^{3}+\frac{16 S_{13}\left(1-S_{12}\right)}{\left(1+S_{12}\right)} B_{5}^{(1)} \eta^{2}-\frac{16 S_{44}}{\alpha\left(1+S_{12}\right)} B_{6}^{(1)} \eta^{2}\right.\right. \\
\left.\left.\quad+A_{17}^{(1)}+A_{18}^{(1)} \eta\right] e^{-\alpha \eta}-4 S_{13} A_{15}^{(1)} \eta-4 S_{13} A_{16}^{(1)}-\frac{2 S_{44}}{\alpha} A_{15}^{(1)}\right\}
\end{array}\right.
$$


where $A_{13}^{(1)}, A_{14}^{(1)}, A_{15}^{(1)}, A_{16}^{(1)}, A_{17}^{(1)}, A_{18}^{(1)}, B_{5}^{(1)}, B_{6}^{(1)}, C_{13}^{(1)}, C_{14}^{(1)}, C_{15}^{(1)}, C_{16}^{(1)}, C_{17}^{(1)}$, and $C_{18}^{(1)}$ are integral constants of the first-order perturbation to $D_{15}$. By using boundary conditions (48) and (49), these constants are determined as

$$
\left\{\begin{array}{l}
A_{13}^{(1)}=A_{14}^{(1)}=A_{15}^{(1)}=A_{16}^{(1)}=A_{17}^{(1)}=A_{18}^{(1)}=B_{5}^{(1)}=B_{6}^{(1)}=0, \\
C_{13}^{(1)}=\frac{1}{1-e^{\alpha}}\left[\frac{4 e^{\alpha / 2}}{\alpha\left(1+S_{12}\right)} B_{2}^{(0)}+\frac{4 e^{\alpha / 2}}{\left(1+S_{12}\right)} B_{1}^{(0)}+\frac{\left(e^{\alpha}-1\right)}{4} A_{3}^{(0)}\right], \\
C_{14}^{(1)}=0, \\
C_{15}^{(1)}=\frac{\left(e^{\alpha}-1\right)}{4\left(1-e^{\alpha}\right)}\left\{\left[\frac{32}{3 \alpha^{2} \lambda_{33}\left(1+S_{12}\right)} B_{2}^{(0)}+\frac{64}{\alpha^{2}\left(1+S_{12}\right)} B_{2}^{(0)}+\frac{64}{\alpha\left(1+S_{12}\right)} B_{1}^{(0)}\right.\right. \\
\left.\left.\quad+4 C_{16}^{(1)}\right] e^{\alpha / 2}-\left(\frac{8 A_{3}^{(0)}+32 C_{13}^{(1)}}{\alpha^{2} \lambda_{33}}\right)\left(1+e^{\alpha}\right)+\left(\frac{4 A_{3}^{(0)}}{\alpha}+\frac{16 A_{3}^{(0)}+64 C_{13}^{(1)}}{\alpha^{3} \lambda_{33}}\right)\left(e^{\alpha}-1\right)\right\}, \\
C_{16}^{(1)}=\frac{1}{1-e^{\alpha}}\left\{\left[-\frac{32}{\alpha^{3} \lambda_{33}\left(1+S_{12}\right)} B_{2}^{(0)}-\frac{32}{\alpha^{2} \lambda_{33}\left(1+S_{12}\right)} B_{1}^{(0)}\right]\left(1+e^{\alpha}\right)\right. \\
\left.\quad+\left[-\frac{8 \alpha^{2}-128}{\alpha^{4} \lambda_{33}\left(1+S_{12}\right)} B_{2}^{(0)}+\frac{64}{\alpha^{3} \lambda_{33}\left(1+S_{12}\right)} B_{1}^{(0)}\right]\left(1-e^{\alpha}\right)\right\}, \\
C_{18}^{(1)}=\frac{1}{\alpha \lambda_{33}\left(1-e^{\alpha}\right)}\left\{\frac{16 A_{3}^{(0)}+64 C_{13}^{(1)}}{\alpha^{2} \lambda_{33}} e^{\alpha / 2}-\left[\frac{16}{3 \alpha^{2} \lambda_{33}\left(1+S_{12}\right)} B_{2}^{(0)}+\frac{32}{\alpha^{2}\left(1+S_{12}\right)} B_{2}^{(0)}\right.\right. \\
\left.+\frac{32}{\alpha\left(1+S_{12}\right)} B_{1}^{(0)}+2 C_{16}^{(1)}\right]\left(1+e^{\alpha}\right)+\left[-\frac{64}{\alpha^{3} \lambda_{33}\left(1+S_{12}\right)} B_{2}^{(0)}-\frac{16}{\alpha^{2} \lambda_{33}\left(1+S_{12}\right)} B_{1}^{(0)}\right. \\
-\frac{8}{\alpha\left(1+S_{12}\right)} B_{2}^{(0)}+\frac{768}{\alpha^{5} \lambda_{33}\left(1+S_{12}\right)} B_{2}^{(0)}+\frac{128}{\alpha^{4} \lambda_{33}\left(1+S_{12}\right)} B_{1}^{(0)}+\frac{128}{\alpha^{3}\left(1+S_{12}\right)} B_{2}^{(0)} \\
\left.\left.+\frac{64}{\alpha^{2}\left(1+S_{12}\right)} B_{1}^{(0)}+\frac{4}{\alpha} C_{16}^{(1)}\right]\left(1-e^{\alpha}\right)\right\},
\end{array} .\right.
$$

Note that there is also no need to determine $C_{17}^{(1)}$.

\section{References}

1. Rao, S.S.; Sunar, M. Piezoelectricity and its use in disturbance sensing and control of flexible structures: A survey. Appl. Mech. Rev. 1994, 47, 113-123. [CrossRef]

2. Pohanka, M. Overview of piezoelectric biosensors, immunosensors and DNA sensors and their applications. Materials 2018, 11, 448. [CrossRef] [PubMed]

3. Soltanrezaee, M.; Bodaghi, M.; Farrokhabadi, A.; Hedayati, R. Nonlinear stability analysis of piecewise actuated piezoelectric microstructures. Int. J. Mech. Sci. 2019, 160, 200-208. [CrossRef]

4. Singh, S.; Sharma, K.; Bui, T.Q. New analytical solutions for modified polarization saturation models in piezoelectric materials. Meccanica 2019, 54, 2443-2459. [CrossRef]

5. Wu, C.C.M.; Kahn, M.; Moy, W. Piezoelectric ceramics with functional gradients: A new application in material design. J. Am. Ceram. Soc. 1996, 79, 809-812. [CrossRef]

6. Shelley, W.F., II; Wan, S.; Bowman, K.J. Functionally-graded piezoelectric ceramics. Mater. Sci. Forum 1999, 308-311, 515-520. [CrossRef]

7. Taya, M.; Almajid, A.A.; Dunn, M.; Takahashi, H. Design of bimorph piezo-composite actuators with functionally-graded microstructure. Sens. Actuators A 2003, 107, 248-260. [CrossRef]

8. Huang, D.J.; Ding, H.J.; Chen, W.Q. Piezoelasticity solutions for functionally-graded piezoelectric beams. Smart Mater. Struct. 2007, 16, 687-695. [CrossRef]

9. Zhong, Z.; Yu, T. Electroelastic analysis of functionally-graded piezoelectric material beam. J. Intell. Mater. Syst. Struct. 2008, 19, 707-713. [CrossRef]

10. Bodaghi, M.; Damanpack, A.R.; Aghdam, M.M.; Shakeri, M. Geometrically non-linear transient thermo-elastic response of FG beams integrated with a pair of FG piezoelectric sensors. Compos. Struct. 2014, 107, 48-59. [CrossRef]

11. Yao, R.X.; Shi, Z.F. Steady-State forced vibration of functionally-graded piezoelectric beams. J. Intell. Mater. Syst. Struct. 2011, 22, 769-779. [CrossRef]

12. He, X.T.; Wang, Y.Z.; Shi, S.J.; Sun, J.Y. An electroelastic solution for functionally-graded piezoelectric material beams with different moduli in tension and compression. J. Intell. Mater. Syst. Struct. 2018, 29, 1649-1669. [CrossRef] 
13. Shakeri, M.; Mirzaeifar, R. Static and dynamic analysis of thick functionally-graded plates with piezoelectric layers using layerwise finite element model. Mech. Adv. Mater. Struct. 2009, 16, 561-575. [CrossRef]

14. Ebrahimi, F. Analytical investigation on vibrations and dynamic response of functionally-graded plate integrated with piezoelectric layers in thermal environment. Mech. Adv. Mater. Struct. 2013, 20, 854-870. [CrossRef]

15. Kulikov, G.M.; Plotnikova, S.V. An analytical approach to three-dimensional coupled thermoelectroelastic analysis of functionally-graded piezoelectric plates. J. Intell. Mater. Syst. Struct. 2017, 28, 435-450. [CrossRef]

16. Alibeigloo, A. Thermo elasticity solution of functionally-graded, solid, circular, and annular plates integrated with piezoelectric layers using the differential quadrature method. Mech. Adv. Mater. Struct. 2018, 25, 766-784. [CrossRef]

17. Akbari, P.; Asanjarani, A. Semi-analytical mechanical and thermal buckling analyses of 2D-FGM circular plates based on the FSDT. Mech. Adv. Mater. Struct. 2019, 26, 753-764. [CrossRef]

18. Alibeigloo, A. Static analysis of a functionally-graded cylindrical shell with piezoelectric layers as sensor and actuator. Smart Mater. Struct. 2009, 18, 065004. [CrossRef]

19. Mehralian, F.; Beni, Y.T.; Ansari, R. Size dependent buckling analysis of functionally-graded piezoelectric cylindrical nanoshell. Compos. Struct. 2016, 152, 45-61. [CrossRef]

20. Biglar, M.; Mirdamadi, H.R. Configuration optimization of piezoelectric patches attached to functionally-graded shear-deformable cylindrical shells considering spillover effects. J. Intell. Mater. Syst. Struct. 2016, 27, 295-313. [CrossRef]

21. Fang, X.Q.; Zhu, C.S.; Liu, J.X.; Zhao, J. Surface energy effect on nonlinear buckling and postbuckling behavior of functionally-graded piezoelectric cylindrical nanoshells under lateral pressure. Mater. Res. Express 2018, 5, 045017. [CrossRef]

22. Ansari Dezfoli, A.R.; Mehrabian, M.A.; Saffaripour, M.H. Two dimensional temperature distributions in plate heat exchangers: An analytical approach. Mathematics 2015, 3, 1255-1273. [CrossRef]

23. Yazıcı, V.; Muradoğlu, Z. A mathematical model and numerical solution of a boundary value problem for a multi-structure plate. Mathematics 2019, 7, 244. [CrossRef]

24. Van Gorder, R.A. Analytical method for the construction of solutions to the Föppl-von Kármán equations governing deflections of a thin flat plate. Int. J. Non Linear Mech. 2012, 47, 1-6. [CrossRef]

25. Vincent, J.J. The bending of a thin circular plate. Philos. Mag. 1931, 12, 185-196. [CrossRef]

26. Chien, W.Z. Large deflection of a circular clamped plate under uniform pressure. Chin. J. Phys. 1947, 7, 102-113.

27. Hu, H.C. On the large deflection of a circular plate under combined action of uniformly distributed load and concentrated load at the center. Chin. J. Phys. 1954, 10, 383-394.

28. Schmidt, R.; DaDeppo, D.A. A new approach to the analysis of shells, plates and membranes with finite deflections. Int. J. Non Linear Mech. 1974, 9, 409-419. [CrossRef]

29. Hwang, C. Large deflection of circular plate under compound load. Appl. Math. Mech. (Engl. Ed.) 1983, 4, 791-804.

30. Chen, S.L.; Kuang, J.C. The perturbation parameter in the problem of large deflection of clamped circular plates. Appl. Math. Mech. (Engl. Ed.) 1981, 2, 137-154.

31. Chen, S.L. Free-parameter perturbation method. In Proceeding for Celebrating 90th Birthday of Professor Chien Wei-Zang; Zhou, Z.W., Ed.; Shanghai University Press: Shanghai, China, 2003; pp. 35-43.

32. Chen, S.L.; Li, Q.Z. Free-parameter perturbation-method solutions of the nonlinear stability of shallow spherical shells. Appl. Math. Mech. (Engl. Ed.) 2004, 25, 963-970.

33. Nowinski, J.L.; Ismail, I.A. Application of a multi-parameter perturbation method to elastostatics. Dev. Theor. Appl. Mech. 1965, 2, 35-45.

34. Chien, W.Z. Second order approximation solution of nonlinear large deflection problem of Yongjiang Railway Bridge in Ningbo. Appl. Math. Mech. (Engl. Ed.) 2002, 23, 493-506.

35. He, X.T.; Chen, S.L. Biparametric perturbation solution of large deflection problem of cantilever beams. Appl. Math. Mech. (Engl. Ed.) 2006, 27, 453-460. [CrossRef]

36. He, X.T.; Cao, L.; Li, Z.Y.; Hu, X.J.; Sun, J.Y. Nonlinear large deflection problems of beams with gradient: A biparametric perturbation method. Appl. Math. Comput. 2013, 219, 7493-7513. [CrossRef] 
37. He, X.T.; Cao, L.; Sun, J.Y.; Zheng, Z.L. Application of a biparametric perturbation method to large-deflection circular plate problems with a bimodular effect under combined loads. J. Math. Anal. Appl. 2014, 420, 48-65. [CrossRef]

38. He, X.T.; Cao, L.; Wang, Y.Z.; Sun, J.Y.; Zheng, Z.L. A biparametric perturbation method for the Föppl-von Kármán equations of bimodular thin plates. J. Math. Anal. Appl. 2017, 455, 1688-1705. [CrossRef]

39. He, X.T.; Li, X.; Yang, Z.X.; Liu, G.H.; Sun, J.Y. Application of biparametric perturbation method to functionally-graded thin plates with different moduli in tension and compression. ZAMM J. Appl. Math. Mech. 2019, 99, e201800213. [CrossRef]

40. Fallah, F.; Nosier, A.; Sharifi, M.; Ghezelbash, F. On perturbation method in mechanical, thermal and thermo-mechanical loadings of plates: Cylindrical bending of FG plates. ZAMM J. Appl. Math. Mech. 2016, 96, 217-232. [CrossRef]

41. Lian, Y.S.; He, X.T.; Shi, S.J.; Yang, Z.X.; Sun, J.Y. A multi-parameter perturbation solution for functionally-graded piezoelectric cantilever beams under combined loads. Materials 2018, 11, 1222. [CrossRef]

42. Lian, Y.S.; He, X.T.; Liu, G.H.; Sun, J.Y.; Zheng, Z.L. Application of perturbation idea to well-known Hencky problem: A perturbation solution without small-rotation-angle assumption. Mech. Res. Commun. 2017, 83, 32-46. [CrossRef]

43. Ding, H.J.; Xu, B.H. General solutions of axisymmetric problems in transversely isotropic body. Appl. Math. Mech. (Engl. Ed.) 1998, 9, 143-151.

44. Ding, H.J.; Li, X.Y.; Chen, W.Q. Analytical solutions for a uniformly loaded circular plate with clamped edges. J. Zhejiang Univ. Science A 2005, 6, 1163-1168.

45. He, X.T.; Yang, Z.X.; Jing, H.X.; Sun, J.Y. One-dimensional theoretical solution and two-dimensional numerical simulation for functionally-graded piezoelectric cantilever beams with different properties in tension and compression. Polymers 2019, 11, 1728.

(C) 2020 by the authors. Licensee MDPI, Basel, Switzerland. This article is an open access article distributed under the terms and conditions of the Creative Commons Attribution (CC BY) license (http://creativecommons.org/licenses/by/4.0/). 